\title{
Epithelial-to-mesenchymal transition drives a pro-metastatic Golgi compaction process through scaffolding protein PAQR11
}

\author{
Xiaochao Tan, ${ }^{1}$ Priyam Banerjee, ${ }^{1}$ Hou-Fu Guo, ${ }^{1}$ Stephen Ireland, ${ }^{2}$ Daniela Pankova, ${ }^{1}$ Young-ho Ahn, ${ }^{3}$ Irodotos Michail Nikolaidis, \\ Xin Liu, ${ }^{1}$ Yanbin Zhao, ${ }^{5}$ Yongming Xue, ${ }^{1}$ Alan R. Burns, ${ }^{6}$ Jonathon Roybal, ${ }^{1}$ Don L. Cibbons, ${ }^{17}$ Tomasz Zal, ${ }^{8}$ Chad J. Creighton, ${ }^{9,10}$ \\ Daniel Ungar, ${ }^{4}$ Yanzhuang Wang, ${ }^{2}$ and Jonathan M. Kurie ${ }^{1}$

\begin{abstract}
'Department of Thoracic/Head and Neck Medical Oncology, The University of Texas MD Anderson Cancer Center, Houston, Texas, USA. 'Department of Molecular, Cellular, and Developmental Biology, University of Michigan, Ann Arbor, Michigan, USA. ${ }^{3}$ Department of Molecular Medicine and Tissue Injury Defense Research Center, Ewha Womans University School of Medicine, Seoul, South Korea. ${ }^{4}$ Department of Biology, University of York, York, United Kingdom. ${ }^{5}$ Department of Internal Medical Oncology, Harbin Medical University Cancer Hospital, Harbin, Heilongjiang Province, China. ${ }^{6}$ College of Optometry, University of Houston, Houston, Texas, USA. . Department of Molecular and Cellular Oncology, ${ }^{8}$ Department of Immunology, and ${ }^{9}$ Department of Bioinformatics and Computational Biology, The University of Texas MD Anderson Cancer Center, Houston, Texas, USA. ${ }^{10}$ Department of Medicine and Dan L Duncan Cancer Center, Baylor College of Medicine, Houston, Texas, USA.
\end{abstract}

\begin{abstract}
Tumor cells gain metastatic capacity through a Golgi phosphoprotein 3-dependent (GOLPH3-dependent) Golgi membrane dispersal process that drives the budding and transport of secretory vesicles. Whether Golgi dispersal underlies the prometastatic vesicular trafficking that is associated with epithelial-to-mesenchymal transition (EMT) remains unclear. Here, we have shown that, rather than causing Golgi dispersal, EMT led to the formation of compact Golgi organelles with improved ribbon linking and cisternal stacking. Ectopic expression of the EMT-activating transcription factor ZEB1 stimulated Golgi compaction and relieved microRNA-mediated repression of the Golgi scaffolding protein PAQR11. Depletion of PAQR11 dispersed Golgi organelles and impaired anterograde vesicle transport to the plasma membrane as well as retrograde vesicle tethering to the Golgi. The N-terminal scaffolding domain of PAQR11 was associated with key regulators of Golgi compaction and vesicle transport in pull-down assays and was required to reconstitute Golgi compaction in PAQR11-deficient tumor cells. Finally, high PAQR11 levels were correlated with EMT and shorter survival in human cancers, and PAQR11 was found to be essential for tumor cell migration and metastasis in EMT-driven lung adenocarcinoma models. We conclude that EMT initiates a PAQR11-mediated Golgi compaction process that drives metastasis.
\end{abstract}

\section{Introduction}

Vesicle-mediated transport of intracellular proteins drives the formation of actin-based membrane projections such as lamellipodia and filopodia and the secretion of extracellular matrix-modifying factors that facilitate angiogenesis and tumor cell invasion (1). Vesicle transport is regulated by the Golgi apparatus, which posttranslationally modifies, sorts, and packages proteins into vesicles that bud from the Golgi and are transported along microtubules to specific intracellular locations (2). Vesicle budding is enhanced by F-actinmediated tensile forces exerted on Golgi membranes through a Golgi phosphoprotein 3 (GOLPH3)/MYO18A bridge (3). GOLPH3 is recruited to the Golgi by a PITPNC1/RAB1B-containing protein complex that binds to Golgi-enriched phosphatidylinositol-4phosphate (4). High expression of GOLPH3 and PITPNC1 promotes metastasis in experimental models and predicts a shorter duration of survival in cancer patients (4). On the basis of these findings, a model has been proposed in which pro-metastatic vesicle trafficking is driven by mechanical forces that promote Golgi dispersal (5).

Authorship note: X. Tan and P. Banerjee contributed equally to this work. Conflict of interest: The authors have declared that no conflict of interest exists. Submitted: May 23, 2016; Accepted: October 6, 2016.

Reference information: / Clin Invest. 2017;127(1):117-131. doi:10.1172/JCI88736.
In certain epithelial tumor types, the ability to shift reversibly between epithelial and mesenchymal states is essential for metastasis (6). This reversible differentiation switch is regulated by mutual antagonism between transcription factors that promote epithelial-to-mesenchymal transition (EMT) (e.g., ZEB, SNAIL, and TWIST family members) and microRNAs (miRs) that target the EMT-activating transcription factors (e.g., miR-200 family members and miR-34a) (6). Epithelial cell polarization along an apicobasal axis causes Golgi vesicles to coalesce into compact ribbon structures that face the apical surface and direct vesicle trafficking toward apical and basolateral membrane compartments (7). During EMT, the Golgi is repositioned to direct vesicle trafficking toward the leading edge of the cell and facilitate the formation of promigratory focal adhesions and cytoplasmic protrusions (8). Thus, the Golgi is a dynamic organelle that is capable of sustained vesicle trafficking when dispersed or compact. However, the way in which EMT-induced vesicular trafficking is regulated remains unclear.

Here, we addressed this question using lung adenocarcinoma cell lines isolated from mice that develop metastatic lung adenocarcinoma from expression of K-ras ${ }^{\mathrm{G} 12 \mathrm{D}}$ and $\mathrm{p} 53^{\mathrm{R} 172 \mathrm{H}}$ (hereafter termed KP cells); these cells have variable metastatic activity that is tightly linked to EMT status and the ZEB1/miR-200 axis $(9,10)$. Highly metastatic KP cells have mesenchymal properties 
A

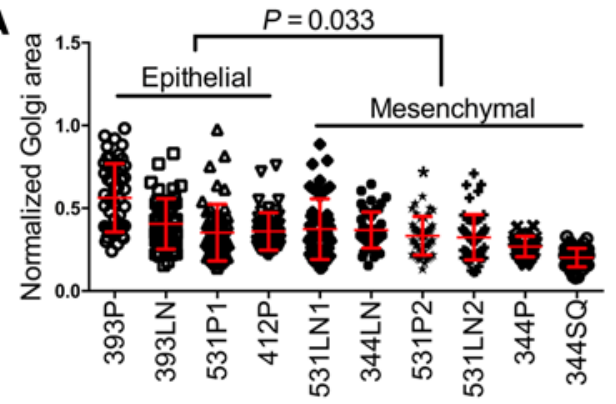

B

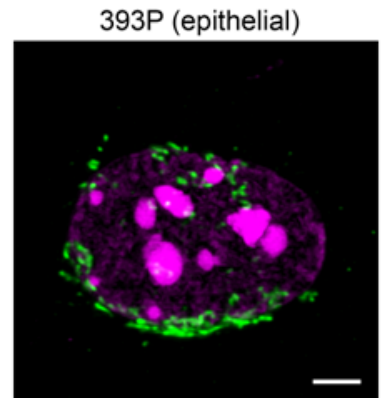

344SQ (mesenchymal)

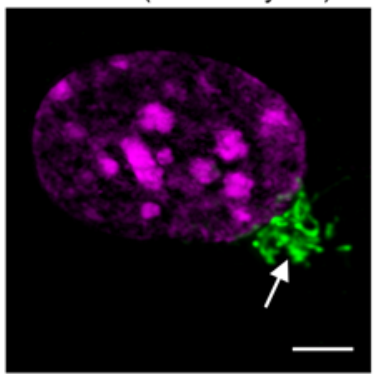

GM130 DAPI
C

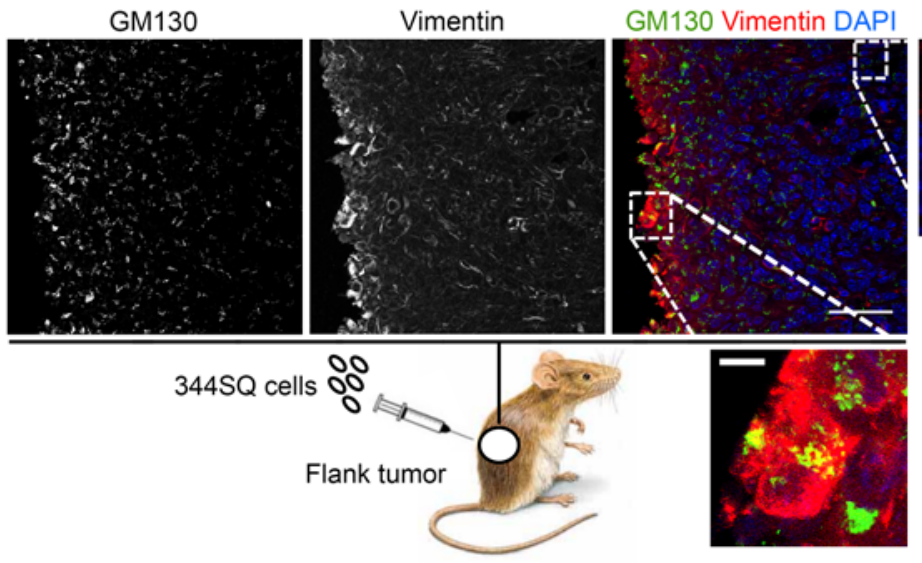

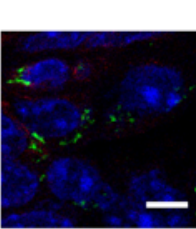

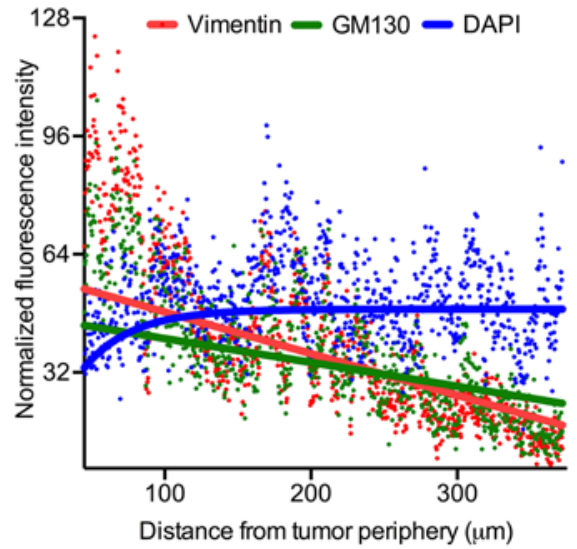

D
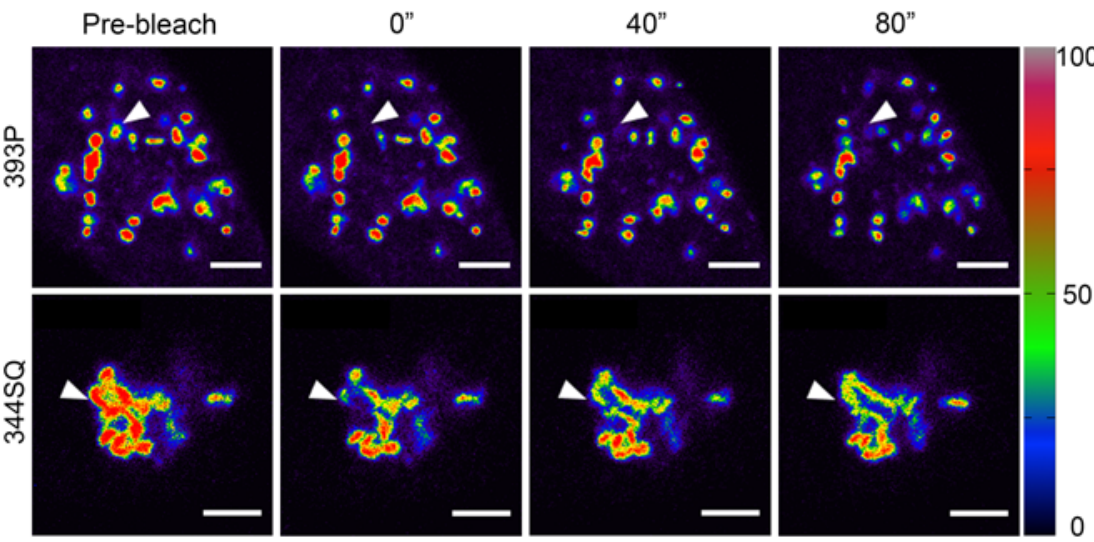

$344 S Q$

F

$393 \mathrm{P}$
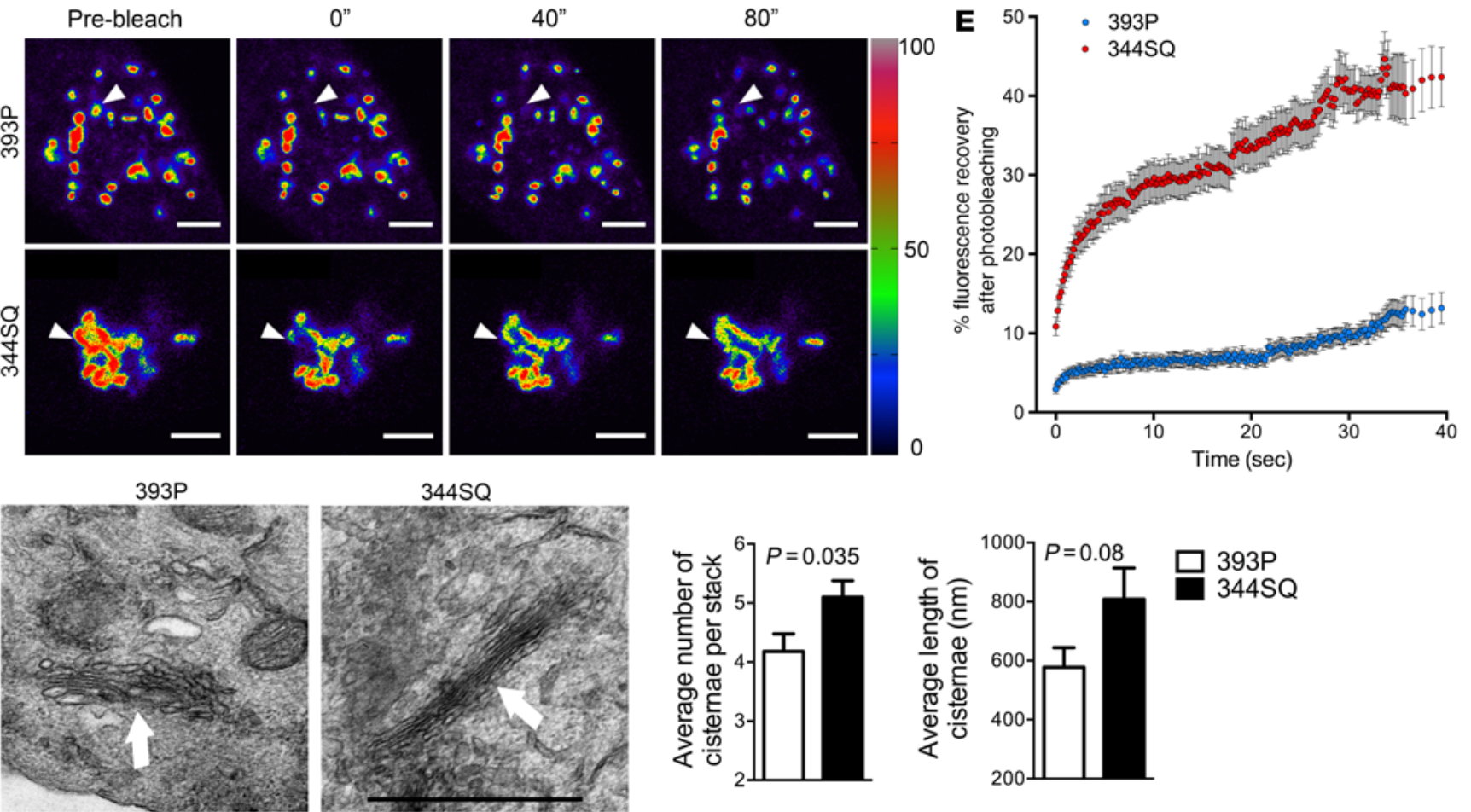
Figure 1. EMT leads to the formation of compact Golgi with improved ribbon linking and cisternal stacking. (A) The scatter plot shows Golgi organelle areas normalized to nuclear areas in epithelial and mesenchymal KP cells. Each dot represents Golgi organelle area in a single cell. (B) Confocal micrographs of Golgi (GM130, green) and nuclei (DAPI, magenta) in representative epithelial (393P) and mesenchymal (344SQ) KP cells. A compact Golgi structure is indicated (arrow). Scale bars: $3 \mu \mathrm{m}$. (C) Confocal micrographs of a flank tumor formed by subcutaneous injection of 344SQ cells into a syngeneic, immunocompetent mouse. Nuclei were counterstained with DAPI (blue) and merged with GM130 (green) and vimentin (red) in an overlaid image (right); the magnified images show compact and dispersed Golgi morphologies in areas of high and low vimentin, respectively. Scale bars: $30 \mu \mathrm{m}$. The scatter plot to the right of the images shows normalized fluorescence intensities of cells (dots) measured radially inward from the tumor periphery. The curve fits for vimentin, GM130, and DAPI (red, green, and blue lines, respectively) were obtained from nonlinear regression using a 1-phase exponential decay equation. (D) Pseudocolored images of the Golgi enzyme GaINAcT in 344SQ cells and 393P cells at indicated time points during FRAP assays. The bleached regions of interest are indicated by arrowheads, and intensity levels are indicated by a lookup table (LUT) bar. Scale bars: $3 \mu \mathrm{m}$. (E) The scatter plot shows the intensity recovery profile (\%) after photobleaching ( $n=20$ cells per group). (F) Electron micrographs of representative cisternal stacks (arrows) in $393 \mathrm{P}$ cells (left) and $344 \mathrm{SQ}$ cells (right). Scale bar: $1 \mu \mathrm{m}$. Bar graphs show the mean numbers of cisternae per stack (left) and cisternal lengths (right). $n \geq 10$ cells per group. $P$ values indicated ( 2 -tailed Student's $t$ test). Results were replicated ( $n \geq 2$ experiments).

and revert to an epithelial state and lose their metastatic activity following ectopic expression of the miR-200b/c/429 cluster; conversely, poorly metastatic KP cells have epithelial properties and undergo mesenchymal differentiation and gain metastatic activity following ectopic expression of the EMT-activating transcription factor ZEB1 $(9,10)$. The findings presented here support a model in which EMT drives metastasis through a Golgi compaction process that is distinct from GOLPH3-dependent mechanical forces that promote metastasis through Golgi dispersal.

\section{Results}

EMT drives Golgi compaction. To determine whether Golgi structural features are regulated during EMT, we initially quantified Golgi organelle areas in a panel of KP cell lines and human lung and breast cancer cell lines classified previously as mesenchymal or epithelial $(10,11)$. As measured from volume projections of cells stained with antibodies against the cis-Golgi marker GM130, Golgi organelle areas were correlated inversely with mesenchymal classification (Figure 1A and Supplemental Figure $1 \mathrm{~A}$; supplemental material available online with this article; doi:10.1172/JCI88736DS1). Compared with mesenchymal 344SQ cells, epithelial 393P cells had less compact Golgi organelles that contained smaller and more numerous Golgi elements, indicative of Golgi element fragmentation (Figure 1B, Supplemental Figure 1B, and Supplemental Video 1). Additionally, Golgi organelle areas were correlated inversely with migratory and metastatic activities in KP cell lines and human lung cancer cell lines (Supplemental Figures 2 and 3).

On the basis of these findings, we sought to determine whether EMT causes Golgi compaction. Indeed, treatment of 393P cells with TGF- $\beta$, which induces KP cells to undergo a partial EMT (10), decreased Golgi organelle areas (Supplemental Figure 4A), whereas shRNA-mediated depletion of E-cadherin dispersed 393P cells but had no detectable effect on Golgi organelle areas (Supplemental Figure 4, B and C), suggesting that the TGF- $\beta-$ induced Golgi compaction was not simply due to a loss of cell-cell contact. Next, we measured Golgi areas in 344SQ cells and A549 human lung cancer cells that were subjected to wound healing assays. Golgi structures were more compact at the wound edge, where cells typically undergo EMT before migrating into the wound (2), than they were in cells located away from the wound edge (Supplemental Figure 5A). Furthermore, Golgi organelles that faced lamellipodia were more compact than Golgi that were not positioned in that direction (Supplemental Figure 5B). Last- ly, we assessed Golgi compaction in flank tumors and orthotopic lung tumors generated by injection of $344 \mathrm{SQ}$ cells into syngeneic, immunocompetent mice. Coimmunofluorescence staining was performed on the tumors to quantify GM130 and vimentin levels, which are correlated with Golgi organelle compaction (12) and EMT, respectively. Vimentin and GM130 staining intensities were highest on the tumor periphery and decreased exponentially with increasing distance from the periphery, whereas cell density remained constant (Figure 1C and Supplemental Figure 6). Measured radially from the tumor periphery inward, vimentin and GM130 staining intensities were significantly correlated (Spearman's rank correlation coefficients $=0.85$ and 0.98 in the flank and orthotopic lung tumors, respectively). Thus, EMT led to the formation of compact Golgi organelles.

The formation of ribbon-linked cisternal stacks increases the mobility of proteins within cisternae and facilitates the accurate location and function of enzymes that modify $\mathrm{N}$ - and O-linked oligosaccharides (13). To determine whether EMT is associated with improved ribbon linking and cisternal stacking, we initially performed fluorescence recovery after photobleaching (FRAP) assays using a GFP-tagged Golgi enzyme, $N$-acetylgalactosaminyltransferase (GalNAcT), which showed that recovery was significantly faster in mesenchymal $344 \mathrm{SQ}$ cells than it was in epithelial 393P cells (Figure 1, D and E, and Supplemental Video 2). To determine whether these cells differ with respect to cisternal stacking, we quantified cisternal numbers and lengths per stack, which were higher in 344SQ cells than they were in 393P cells (Figure 1F). Thus, EMT is associated with improved ribbon linking and cisternal stacking.

GOLPH3 levels were similar in KP cells with epithelial or mesenchymal features (Supplemental Figure 7A), suggesting that the EMT-driven Golgi compaction process was not mediated through changes in GOLPH3 expression. Microtubule acetylation facilitates Golgi ministack transport (14), and $\alpha$-tubulin acetylation is increased through an inhibitory interaction between paxillin and the $\alpha$-tubulin deacetylase HDAC6 (15). However, the amounts of acetylated $\alpha$-tubulin and paxillin were similar in KP cells with epithelial or mesenchymal features (Supplemental Figure 7B), arguing against microtubule acetylation as a mechanism by which KP cells regulate Golgi organelle integrity during EMT. In contrast, the levels of ZEB1, a critical driver of EMT and metastasis in KP cells $(9-11,16)$, were negatively correlated with Golgi areas in the KP cell line panel (Supplemental Figure 7C). Ectopic ZEB1 expression in epithelial lung cancer cells (393P, 307P, and HCC827) and 
A

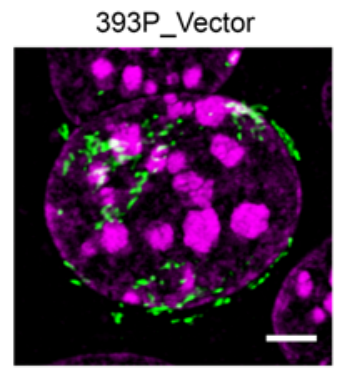

B
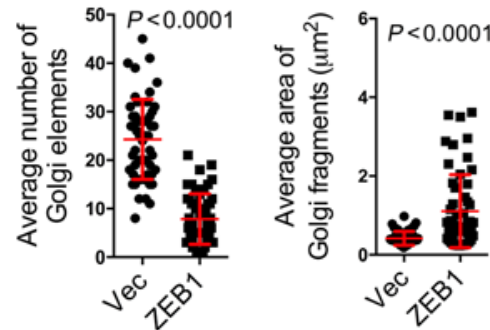

D

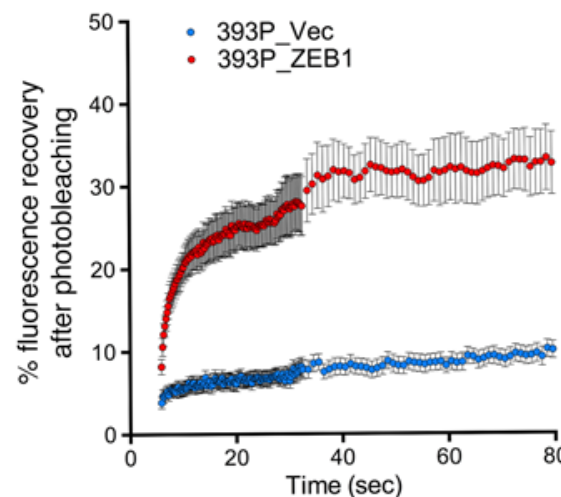

E

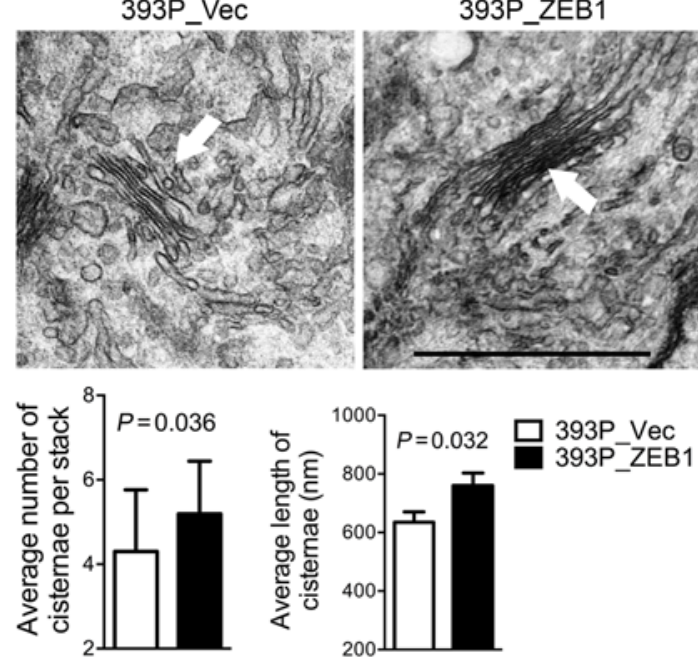

393P_ZEB1

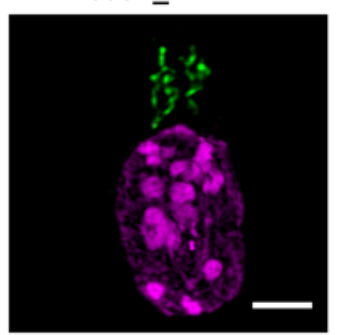

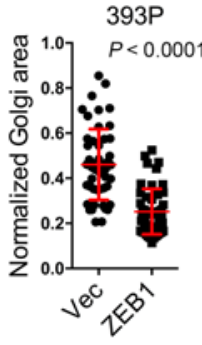

C

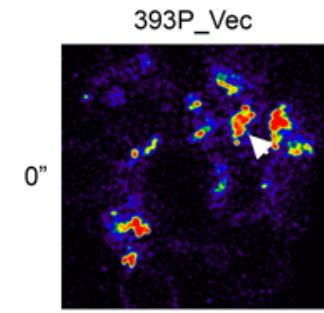

$18 "$
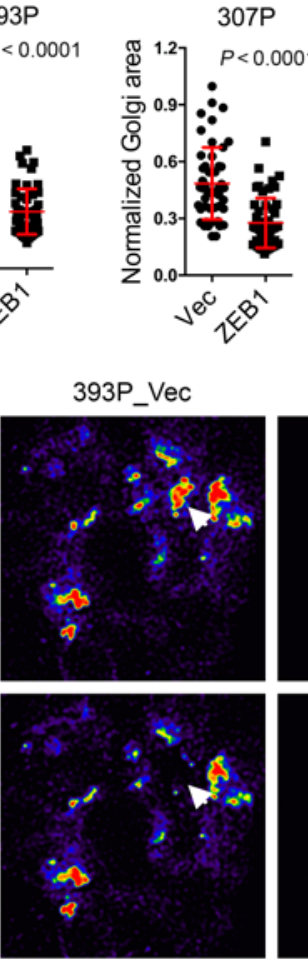

HCC827

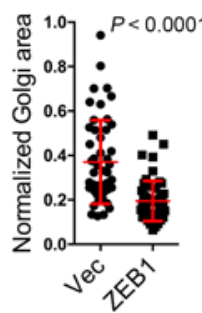

393P_ZEB1
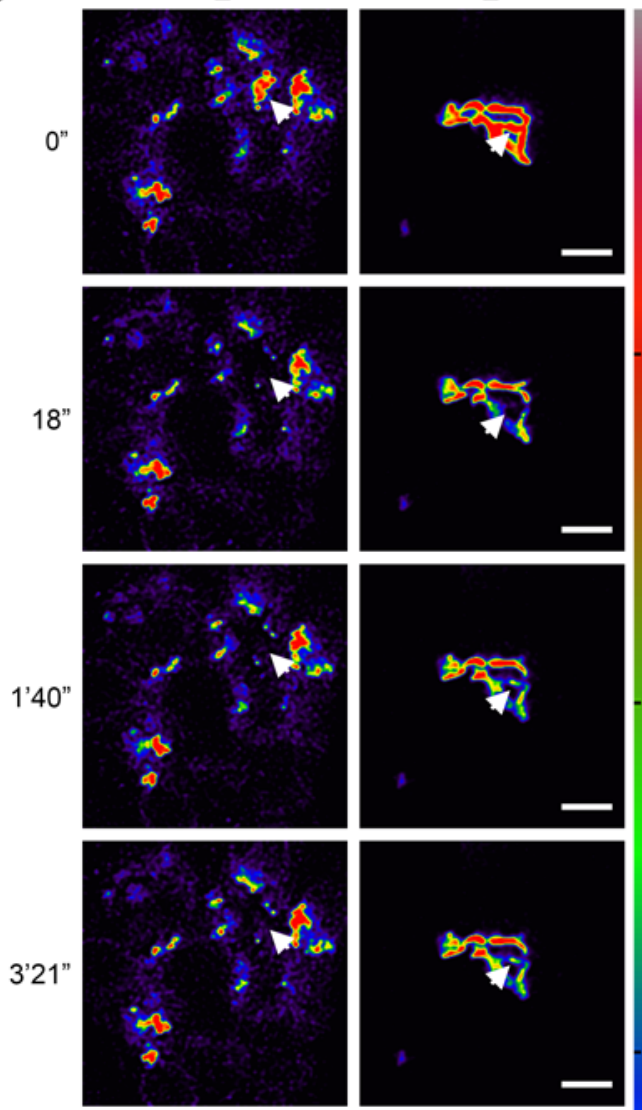

1'40"
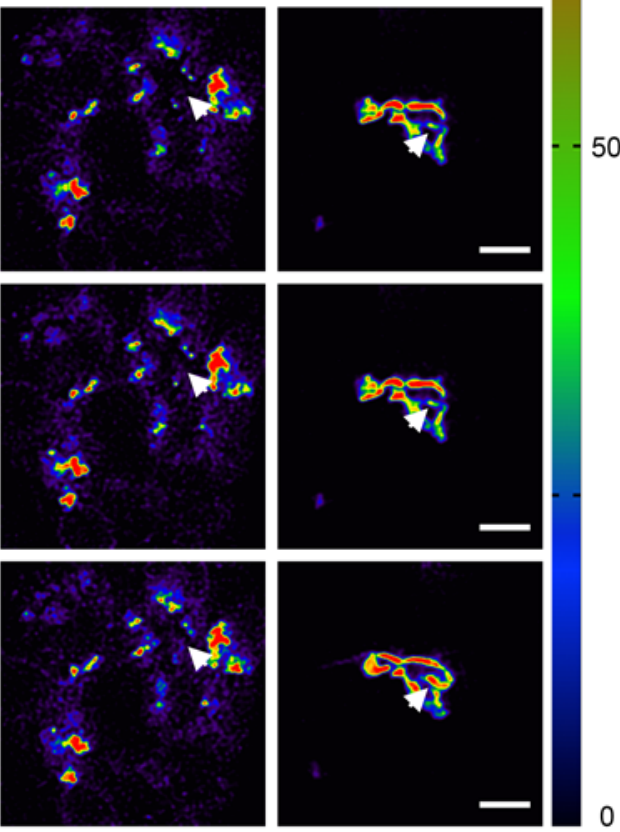

Figure 2. Ectopic ZEB1 expression increases Golgi compaction. (A) Confocal micrographs of Golgi (GM130, green) and nuclei (DAPI, magenta) in 393P cells stably transfected with empty (Vec) or ZEB1 expression vectors. Scale bars: $3 \mu \mathrm{m}$. Scatter plots show the normalized Golgi areas in 393P cells (left), 307P cells (middle), and HCC827 cells (right) stably transfected with ZEB1 or empty (Vec) expression vectors. Each dot represents values from a single cell. (B) Scatter plots show the average Golgi element numbers (left) and areas (right) in 393P cells stably transfected with ZEB1 or empty (Vec) expression vectors. Each dot represents values from a single cell. (C) Pseudocolored images of FRAP assays using the Golgi enzyme GalNAcT in 393P_vector cells (left) and 393P_ZEB1 cells (right) at time points indicated to the left of each row. The bleached regions of interest are indicated by arrowheads, and intensity levels are indicated by LUT bar (right). Scale bars: $3 \mu \mathrm{m}$. (D) The scatter plot shows the intensity recovery profile (\%) after photobleaching. $n=20$ cells per group. (E) Electron micrographs of representative cisternal stacks (arrows) in 393P_vector cells (left) and 393P_ZEB1 cells (right). Scale bar: $1 \mu \mathrm{m}$. Bar graphs show the mean numbers of cisternae per stack (left) and cisternal lengths (right). $n>10$ cells per group. $P$ values were determined using 2 -tailed Student's $t$ test. Results were replicated ( $n \geq 2$ experiments). 
Madin Darby canine kidney (MDCK) cells led to a reduction in Golgi organelle areas (Figure 2A; Supplemental Figure 7, D and E; and Supplemental Video 3) and, in 393P cells, enhanced Golgi element condensation (Figure 2B), Golgi ribbon linking (Figure 2, C and D, and Supplemental Video 4), and cisternal stacking (Figure 2E). Conversely, shRNA-mediated depletion of ZEB1 in 344SQ cells (Supplemental Figure 7F) led to Golgi element fragmentation (Supplemental Figure 7G) and decreased Golgi ribbon linking (Supplemental Figure $7 \mathrm{H}$ ), but Golgi organelles remained compact (Supplemental Figure 7I), which may be related to residual ZEB1 expression in the ZEB1 shRNA transfectants (Supplemental Figure 7F). These findings suggest that Golgi elements initially fuse and subsequently coalesce into a compact organelle through ZEB1-dependent mechanisms.

The Golgi scaffolding protein PAQR11 enables Golgi compaction. To identify candidate mediators of ZEB1-induced Golgi compaction, we mined Affymetrix transcriptional profiles in Gene Expression Omnibus GSE61395 (17), which compares the transcriptomes of 393P cells stably transfected with ZEB1 or empty expression vectors. We found that the Gene Ontology database (http:// geneontology.org/) term "Golgi apparatus" was significantly enriched in genes that were upregulated, but not downregulated, by ZEB1 (data not shown). Of 33 upregulated genes classified as Golgi-related in both the Gene Ontology database and the Universal Protein database (http://www.uniprot.org/), 18 were confirmed to be upregulated ( $\geq 2$-fold, 393P_ZEB1 versus 393P_vector) by quantitative reverse transcriptase PCR (qPCR) analysis (Figure $3 \mathrm{~A}$ and Supplemental Table 1). Proteins encoded by the genes are involved in diverse Golgi-related functions, including vesicular trafficking (RAB38 and SYTL2) (18-20), endomannosidase activity (MANEA) $(21,22)$, G protein-coupled receptor activity (RGS20) (23), lipid transport (ABCA5) (24), and signal transduction (CNKSR2 and PAQR11) $(25,26)$.

A member of a family of progestin and adiponectin $Q$ receptors with 7-transmembrane domains $(27,28)$, PAQR11 (encoded by $M M D$ ) has no homology to known drivers of Golgi compaction such as the motor proteins that transport Golgi elements along microtubules (e.g., KIFs and dyneins) or the adhesive proteins that mediate Golgi cisternal stacking and ribbon linking (e.g., GRASP55 and GRASP65) (29, 30). Instead, PAQR11 functions as a scaffold for multiprotein complexes in the Golgi (27). We reasoned that, because Golgi compaction involves the recruitment and coordinated actions of multiple proteins, the scaffolding functions of PAQR11 may mediate ZEB1-induced Golgi compaction. To address this possibility, we first confirmed that ectopic ZEB1 expression increased PAQR11 protein levels in 393P cells (Supplemental Figure 8A). We then performed time-lapse microscopy on 344SQ cells cotransfected with EGFP-tagged PAQR11 and red fluorescent protein-tagged GalNAcT, which confirmed that PAQR11 is a dedicated Golgi protein (Supplemental Video 5). Next, we depleted PAQR11 in 344SQ cells, 393P_ZEB1 cells, and H1299 human lung cancer cells by stable transfection of PAQR11 shRNAs (Supplemental Figure 8B) and found that PAQR11 deficiency led to Golgi organelle dispersal (Figure 3B), Golgi element fragmentation (Supplemental Figure 8C), and a loss of cisternal ribbon linking (Figure 3, C and D, and Supplemental Video 6) and stacking (Figure 3E). Golgi compaction was restored in PAQR11 shRNA- transfected cells by ectopic PAQR11 expression (Supplemental Figure 8D). Ectopic PAQR11 expression in lung cancer cells with low endogenous PAQR11 levels (393P and H226) led to a reduction in Golgi organelle areas (Supplemental Figure 8E). Thus, PAQR11 is a critical mediator of ZEB1-induced Golgi compaction.

Directional trafficking of secretory vesicles mediates cellular shifts between apico-basal and front-rear axes of polarity $(7,8)$. To determine whether ZEB1 regulates vesicle trafficking through PAQR11, we initially performed vesicular stomatitis virus-G (VSV-G) assays and found that ectopic ZEB1 expression increased anterograde vesicle trafficking to the plasma membrane, which was sharply attenuated by PAQR11 depletion (Figure 4A). Next, we examined whether PAQR11 regulates the tethering of retrograde vesicles to Golgi membranes, an essential step in protein trafficking within the Golgi (31). Golgi membranes were isolated from PAQR11-deficient and -replete HEK293 cells that stably express cyan fluorescent protein-tagged (CFP-tagged) $\beta$-1,4-galactosyltransferase (GalT) and incubated with vesicles containing yellow fluorescent protein-tagged (YFP-tagged) GalT. In this assay, colocalization of Golgi and vesicle particles provides a measure of vesicle tethering efficiency (32). Vesicles colocalized less with PAQR11-deficient Golgi than they did with PAQR11-replete Golgi (Supplemental Figure 9A), suggesting that PAQR11 promotes retrograde vesicle tethering to Golgi membranes. Lastly, we quantified the ability of PAQR11-deficient and -replete 344SQ cells to form lumenized spheres in 3D Matrigel, a measure of apico-basal polarization (10). PAQR11-deficient 344SQ cells formed structures with incomplete lumens and disorganized Golgi (Figure 4B and Supplemental Figure 9B), and lumen formation was restored by reconstitution with full-length but not $\mathrm{N}$ - or C-terminally truncated PAQR11 that did not localize to the Golgi (Figure 4C and Supplemental Figure 9C). Thus, PAQR11 enables ZEB1-driven vesicle trafficking and apico-basal polarization.

To elucidate the way in which PAQR11 regulates Golgi compaction and vesicle trafficking, we identified proteins that bind to the PAQR11 scaffolding domain by performing pull-down assays on 344SQ cell extracts using PAQR11 amino acids 1-28 as bait. On the basis of a prediction algorithm (https://www.predictprotein. org), this N-terminal motif projects into the cytoplasm (Supplemental Figure 10A) and may function as a scaffolding domain similarly to the N-terminus of PAQR3 (33). Protein-binding activity of the PAQR11 N-terminal peptide was greater than that of a control PAQR11 C-terminal peptide (Supplemental Figure 10B). Sequencing of the proteins bound to the N-terminal peptide revealed multiple regulators of Golgi compaction (e.g., DCTN1, DCTN2, and ARF1) and vesicular biogenesis and trafficking (e.g., COPA, COPB1, COPG1, KIF2A, KIFC1, KIF22, SEC24A, and ARF1) (Figure 5A and Supplemental Table 2). On the basis of these findings, we postulated that the scaffolding domain of PAQR11 is essential for Golgi compaction and vesicle trafficking. To test this hypothesis, we reconstituted PAQR11-deficient 344SQ cells with full-length or N-terminally truncated PAQR11 expression constructs. PAQR11 mutants that lack amino acids $1-10(\Delta \mathrm{N} 10)$, $1-20(\Delta \mathrm{N} 20)$, or 1-28 ( $\Delta \mathrm{N} 28)$ did not localize in the Golgi (Supplemental Figure 10C). In contrast, a PAQR11 mutant that lacks amino acids 1-5 ( $\triangle \mathrm{N} 5)$ localized in the Golgi similarly to WT PAQR11 (Supplemental Figure 10, C and D) but failed to rescue Golgi 
A

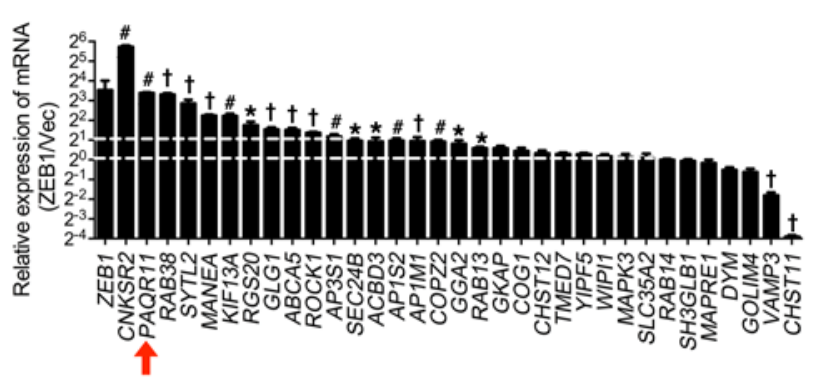

C
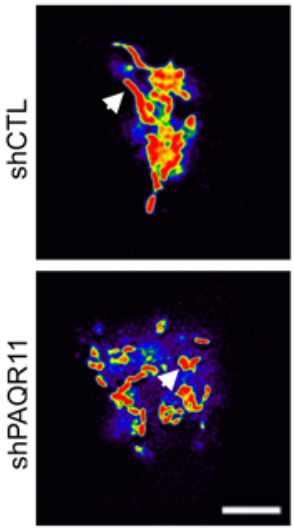

0"
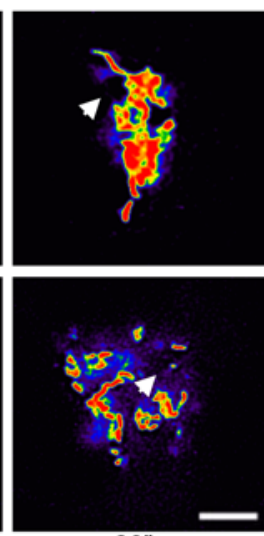

$28 "$
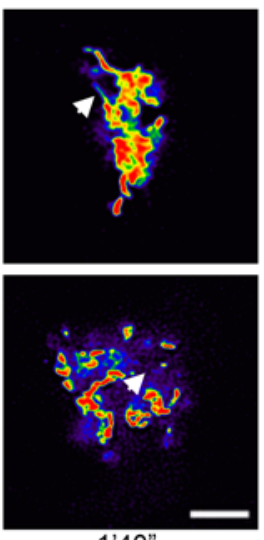
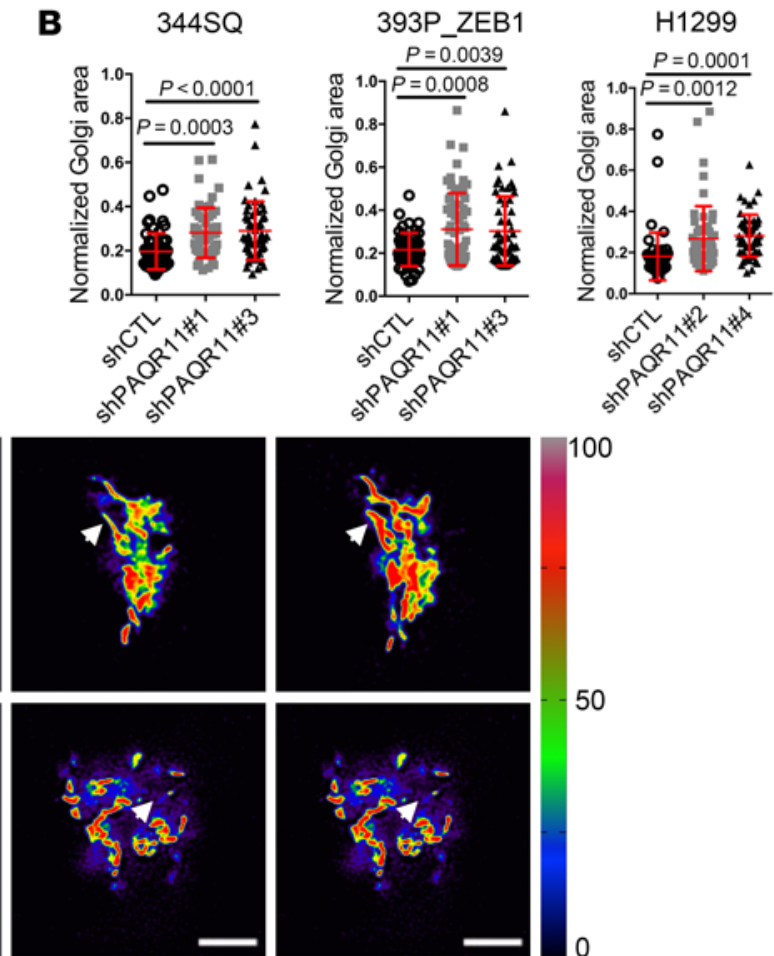

3'20"

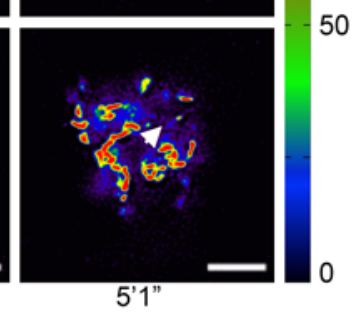

50

E

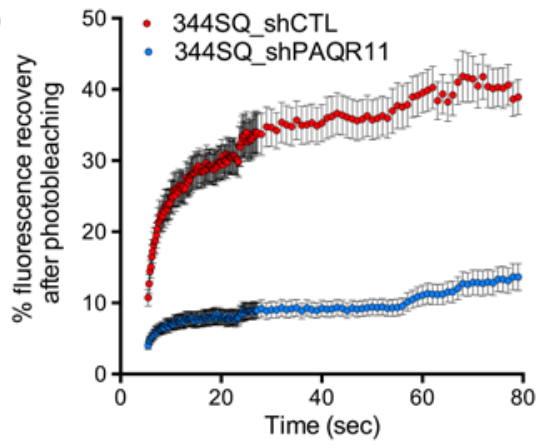
344SQ_shCTL

344SQ_ShPAQR11
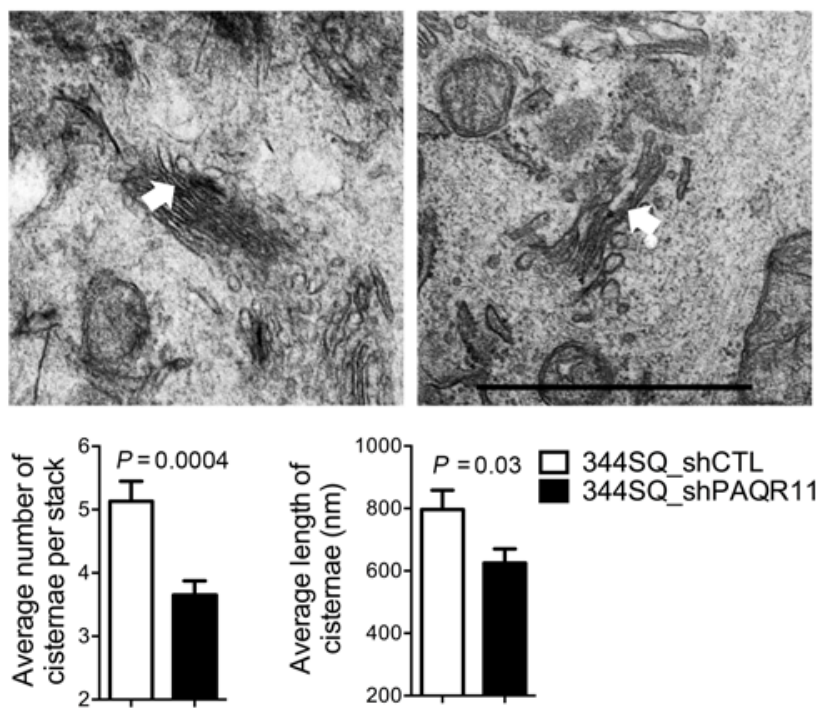

344SQ_shCTL

344SQ_ShPAQR11

100

Figure 3. PAQR11 depletion impairs Golgi compaction. (A) The bar graph shows the results of qPCR analysis performed to validate Affymetrix profiles of Golgi-related genes in 393P_ZEB1 cells and 393P_vector cells $(n=3)$. Expression levels are relative to values in 393P_vector cells, which were set at 1.0 . ${ }^{*} P<0.05$ ${ }^{\#} P<0.01{ }^{\dagger} P<0.001$. (B) Scatter plots show Golgi areas normalized to nuclear areas in 344SQ cells (left), 393P_ZEB1 cells (middle), and H1299 cells (right) stably transfected with 1 of 2 distinct PAQR11 shRNAs (shPAQR11\#1 or shPAQR11\#3) or scrambled control shRNA (shCTL). Each dot represents values from a single cell. (C) Pseudocolored images of the Golgi enzyme GalNAcT during FRAP assays on 344SQ cells stably expressing PAQR11 shRNA (shPAQR11, bottom panels) or scrambled shRNA (shCTL, top panels) at time points indicated under the images. The bleached regions of interest are indicated by arrowheads, and intensity levels are indicated by LUT bars (right). Scale bars: $3 \mu \mathrm{m}$. (D) The scatter plot shows the intensity recovery profile (\%) after photobleaching. $n \geq 20$ cells per group. (E) Electron micrographs of representative cisternal stacks (arrows) in 344SQ_shCTL cells (left) and 344SQ_shPAQR11 cells (right). Scale bar: $1 \mu$ m. Bar graphs show the mean numbers of cisternae per stack (left) and cisternal lengths (right). $n=10$ cells per group. $P$ values were determined using 2 -tailed Student's $t$ test or ANOVA for comparisons between 2 groups or more than 2 groups, respectively. Results were replicated ( $n \geq 2$ experiments).

element condensation (Figure 5B) and Golgi organelle compaction (Figure $5 \mathrm{C}$ ). Collectively, these findings suggest that the $\mathrm{N}$ terminus of PAQR11 has Golgi localization and scaffolding functions that regulate Golgi compaction and vesicle trafficking.
$P A Q R 11$ is a target of ZEB1-silenced miRs. The above findings warranted further studies to elucidate the way in which ZEB1 upregulates PAQR11 expression. Given that ZEB1 functions primarily as a transcriptional repressor (34), we postulated that ZEB1 
A
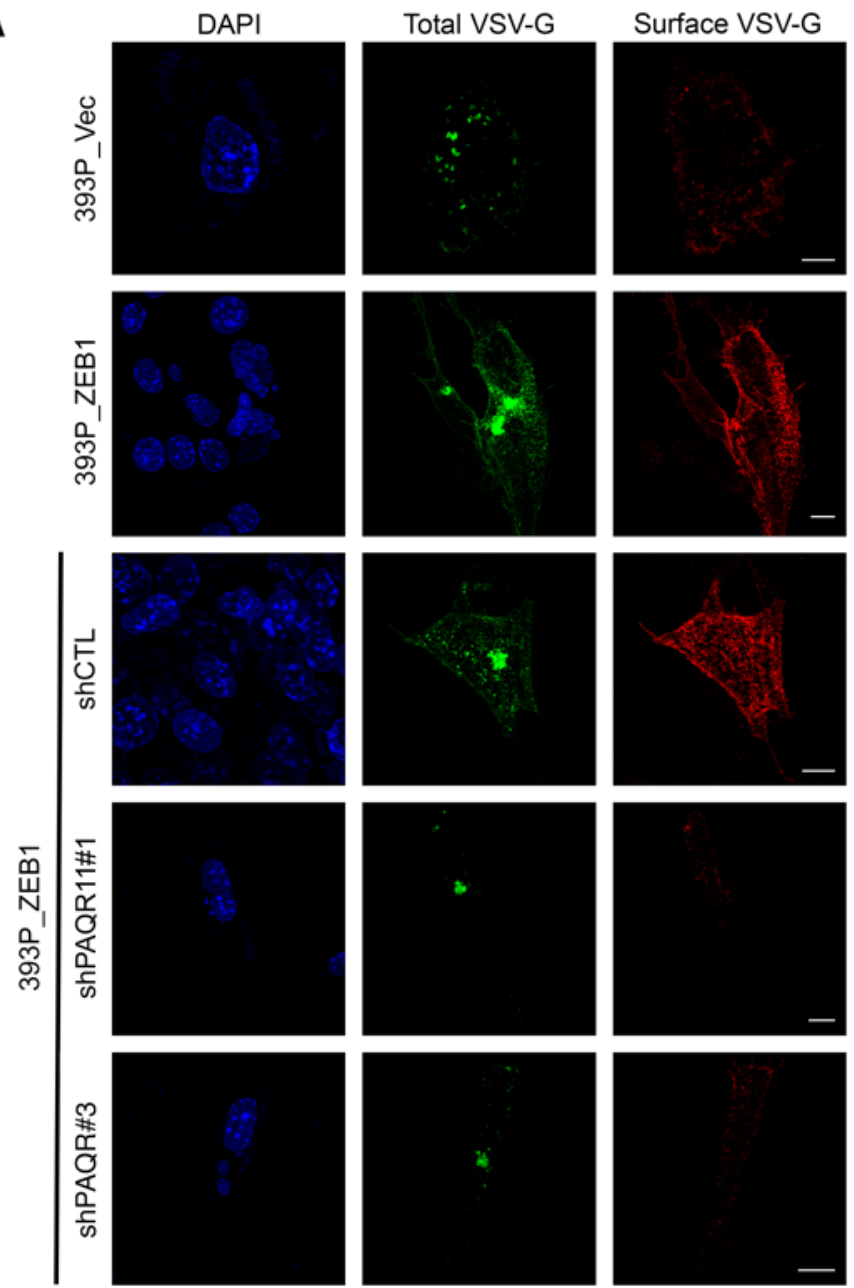

344SQ_shCTL

B
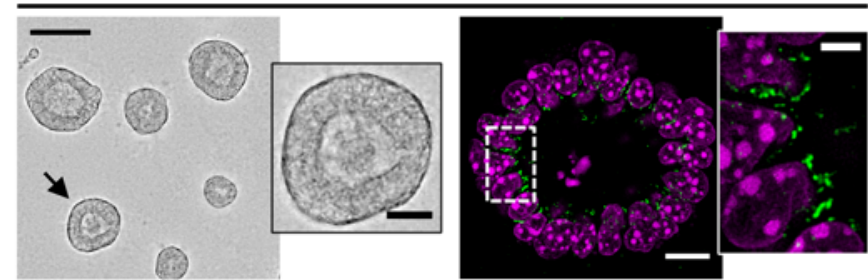

GM130 DAPI

344SQ shPAQR11
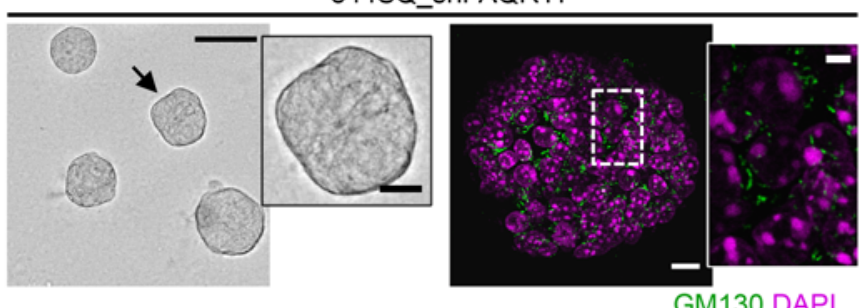
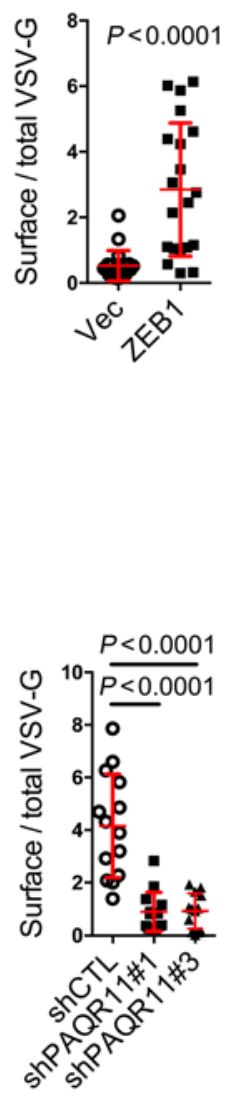

Figure 4. PAQR11 depletion attenuates anterograde vesicle trafficking and impairs cell polarization. (A) Images of EGFP-VSV-G-transfected 393P vector cells (top row), 393P_ZEB1 cells (second row), and 393P_ZEB1 cells stably transfected with scrambled shRNA (shCTL, third row) or PAQR11 shRNA \#1 (fourth row) or \#3 (bottom row) after transfer to the permissive temperature. Total VSV-G was detected by measurement of EGFP signal intensity, and plasma membrane-bound (surface) VSV-G was detected by staining of nonpermeabilized cells with an anti-VSV-G antibody. Scale bars: $10 \mu \mathrm{m}$. The scatter plots display the ratio of surface VSV-G to total VSV-G in each cell (dots, $n>15$ cells per group) for each condition. (B) Micrographs of 344SQ spheres grown in 3D Matrigel cultures. In brightfield images (left), spheres with intact lumens (top) or defective lumens (bottom) are indicated with an arrow and shown at higher magnifications (insets) In confocal images (right), spheres with organized (top) or disorganized (bottom) Golgi alignment along lumen surfaces (boxed areas) are shown at higher magnifications (insets). Scale bars: $75 \mu \mathrm{m}$ and $20 \mu \mathrm{m}$ (insets). (C) Bright-field micrographs of 344SQ spheres showing that lumen formation in PAQR11-deficient structures is rescued by reconstitution with WT PAQR11 (PAQR11) but not truncated PAQR11 lacking $\mathrm{N}$-terminal $(\Delta \mathrm{N})$ or $\mathrm{C}$-terminal $(\Delta \mathrm{C})$ peptides. Scale bars: $40 \mu \mathrm{m}$. $P$ values were determined using 2-tailed Student's $t$ test or ANOVA for comparisons between 2 groups or more than 2 groups, respectively. Results were replicated ( $n \geq 2$ experiments).
C
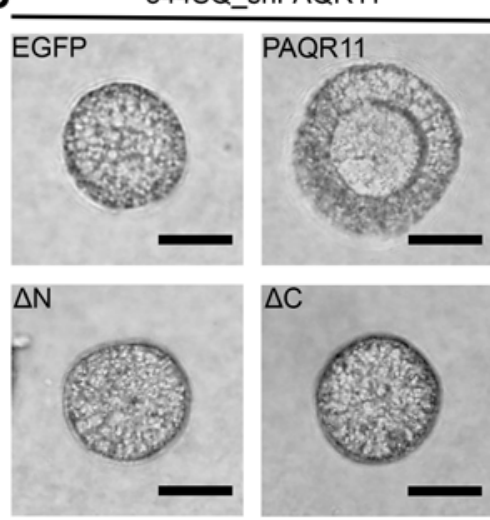

upregulates PAQR11 by silencing miRs that target PAQR11. To test this hypothesis, we examined the PAQR11 3'-UTR and found that it has predicted binding sites for miR-200b/c, -148a, -206, and -182 (TargetScan, www.targetscan.org), the levels of which were correlated inversely with ZEB1 and PAQR11 levels in the KP cell line panel (Figure 6A and Supplemental Table 3). The activity of a PAQR11 3'-UTR reporter was repressed by ectopic miR-200b/c, -148a, or -206 but not -182 (Figure 6B), and miR-induced repression of PAQR11 3'-UTR reporter activity was relieved by mutagenesis of the concordant miR binding sites (Figure 6C). Ectopic expression of each miR (Figure 6D) was sufficient to decrease the levels of PAQR11 mRNA (Figure 6E) and protein (Figure 6F) and 
A

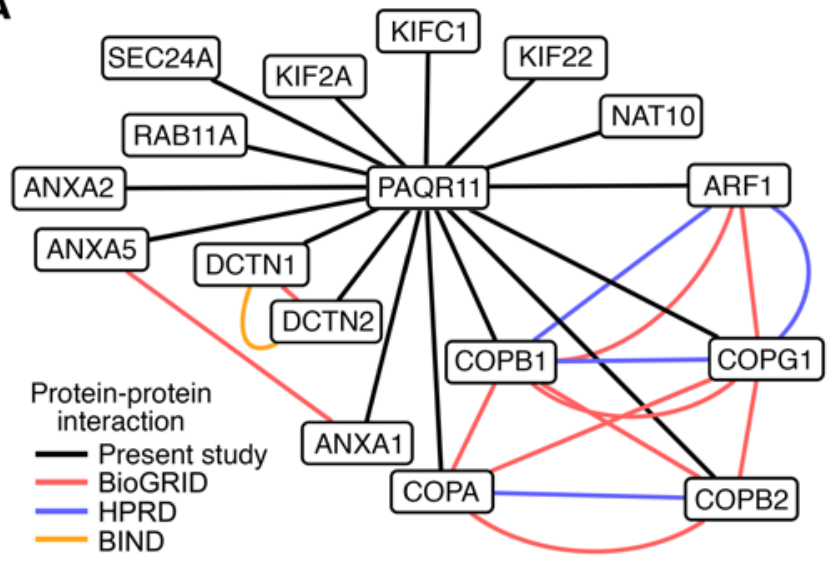

B
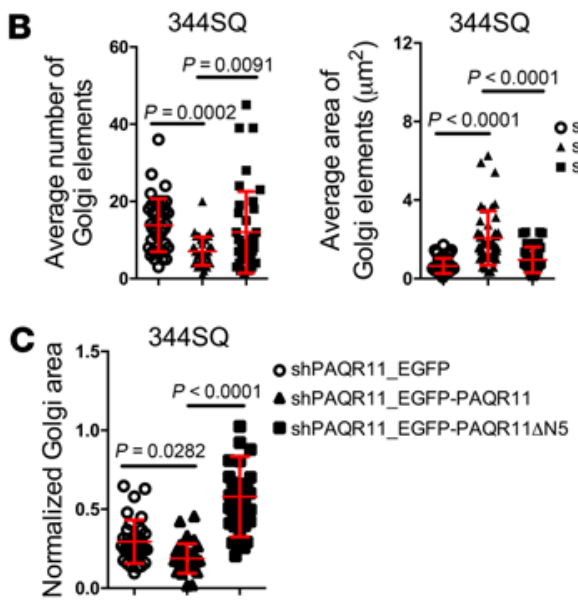

Figure 5. PAQR11 has scaffolding functions required for Golgi compaction. (A) PAQR11-associated proteins identified by liquid chromatographytandem mass spectrometry. Lines indicate known protein-protein interactions as shown here by pull-down assays (present study) and as reported in Biological General Repository for Interaction Data sets (BioGRID), Biomolecular Interaction Network Database (BIND), and Human Protein Reference Database (HPRD). (B) Scatter plots show the average Golgi element number (left) and areas (right) in 344SQ_shPAQR11 cells stably transfected with vectors that express EGFP alone (shPAQR11_EGFP), WT PAQR11 (shPAQR11_EGFP-PAQR11), or mutant PAQR11 that lacks amino acids 1-5 (shPAQR11_EGFP-PAQR11 $\triangle$ N5). Each dot represents values from a single cell. (C) The scatter plot shows Golgi areas normalized to nuclear areas in 344SQ_shPAQR11 cells stably transfected with vectors that express EGFP alone (shPAQR11_EGFP), WT PAQR11 (shPAQR11_EGFP-PAQR11), or mutant PAQR11 that lacks amino acids 1-5 (shPAQR11_EGFP-PAQR11 $\Delta$ N5). Each dot represents values from a single cell. $P$ values were determined using 2-tailed Student's $t$ test or ANOVA for comparisons between 2 groups or more than 2 groups, respectively. Results in $\mathbf{B}$ and $\mathbf{C}$ were replicated ( $n \geq 2$ experiments).

induce Golgi dispersal (Figure 6G), suggesting that PAQR11 is a functional target of miR-200c, miR-148a, and miR-206.

ZEB1 directly silences miR-200c (35), but whether miR-148a and miR-206 are transcriptional targets of ZEB1 is unclear. To address this question, we performed ZEB1 gain- and loss-of-function studies, which showed that all 3 miRs are silenced by ectopic ZEB1 expression (Figure 7A) and upregulated by ZEB1 depletion (Figure 7B). To determine how ZEB1 silences miR-148a, we examined the miR-148a promoter, which revealed 3 predicted ZEB1-binding sites
(TRANSFAC; http://www.gene-regulation.com/pub/databases. $\mathrm{html}$ ). Mutagenesis of these sites increased Mir148a promoter activity in reporter assays (Figure 7C), and ZEB1 bound to these promoter elements in ChIP assays (Figure 7D), suggesting that miR-148a is a direct transcriptional target of ZEB1. Examination of the Mir206 genomic locus revealed predicted binding sites for ZEB1 and GATA3 within the miR-206 precursor. The activity of a reporter containing the miR-206 precursor was repressed by ectopic ZEB1 expression, and ZEB1-induced repression was relieved by mutagenesis of the predicted binding site for GATA3 but not ZEB1 (Figure 7E). In ChIP assays of the miR-206 precursor, binding activity was detected for GATA3 but not ZEB1 (Figure 7F), and shRNA-mediated depletion of GATA3 (Figure 7G), a miR-200 target that is highly expressed in KP cells (16), increased the expression of miR-206 but not miR-148a (Figure 7H), suggesting that GATA3 mediates ZEB1-induced suppression of miR-206. Thus, ZEB1 silences miR-148a and miR-206 through distinct mechanisms.

PAQR11 is essential for EMT-driven metastasis. Kaplan-Meier analysis showed that PAQR11 levels were prognostic for shorter survival durations in a compendium of 12 independent human lung adenocarcinoma cohorts (Supplemental Table 4) and in a pan-cancer analysis of over 30 tumor types available through The Cancer Genome Atlas (Figure 8A). Furthermore, PAQR11 levels were correlated with the presence of an EMT-related gene expression signature (36) in compendia of human lung adenocarcinoma cohorts and breast adenocarcinoma cohorts (Supplemental Table 5). These findings led us to postulate that PAQR11 is required for EMT-driven metastasis. To test this hypothesis, we initially injected PAQR11deficient and -replete $344 \mathrm{SQ}$ cells into syngeneic, immunocompetent mice and found that the flank tumors generated were of similar size, but PAQR11-deficient tumors were markedly less metastatic (Figure 8B and Supplemental Figure 11A). Furthermore, intrathoracic injection of PAQR11-replete or -deficient $\mathrm{H} 1299$ human lung cancer cells into nude mice showed that PAQR11 deficiency reduced orthotopic lung tumor size and the number of metastases to mediastinal lymph nodes and the contralateral lung (Supplemental Figure $11 \mathrm{~B}$ and data not shown). Conversely, compared with empty vector controls, 393P cells that have ectopic PAQR11 expression formed flank tumors that were larger and generated more lung metastases (Supplemental Figure 11, C and D). To determine how PAQR11 enhances metastatic activity, we examined the effect of PAQR11 deficiency on the in vitro properties of KP cells and human lung cancer cells. PAQR11 depletion reduced tumor cell migration in wound healing assays (Figure 8C and Supplemental Figure 12A) and at low density on plastic (Figure 8D and Supplemental Figure 12B). In collagen gels, tumor cells formed spheroids that invaded collectively and as single cells that exhibited ameboid and mesenchymal morphologies (Supplemental Video 7), and PAQR11 depletion inhibited spheroid invasion (Figure 8E and Supplemental Figure 12C). PAQR11 depletion decreased the migratory and invasive activities of tumor cells in Boyden chamber assays (Figure 8F and Supplemental Figure 13). Reconstitution with WT but not $\triangle \mathrm{N} 5$ mutant PAQR11 reversed the effects of PAQR11 shRNAs on cell migration and invasion in Boyden chambers (Supplemental Figure 14, A and B) and single-cell migration on plastic (Supplemental Figure 14, C-F). Conversely, ectopic PAQR11 expression increased the migration and invasion of 393P cells and H441 cells (Supplemental Figure 15). PAQR11- 
A

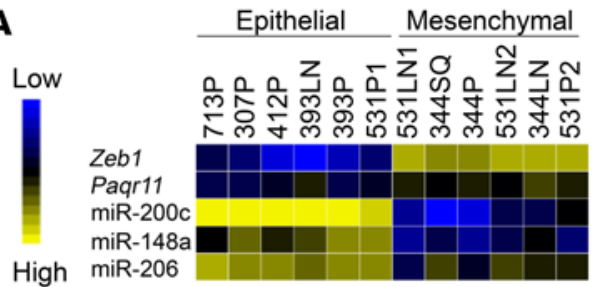

B
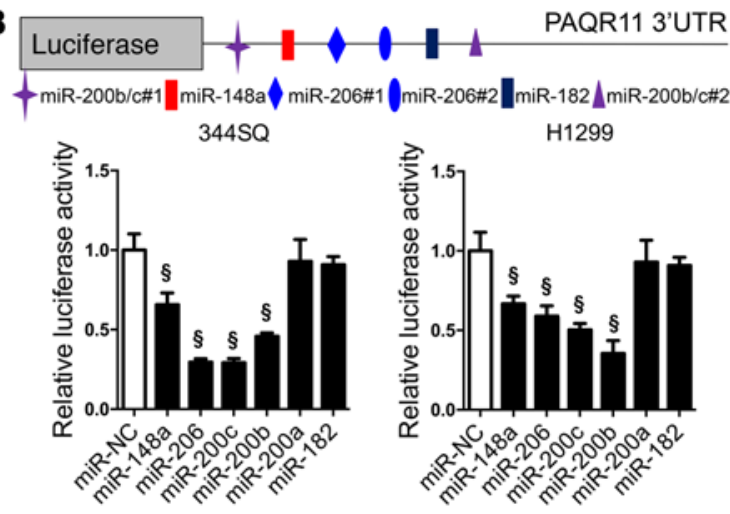

C
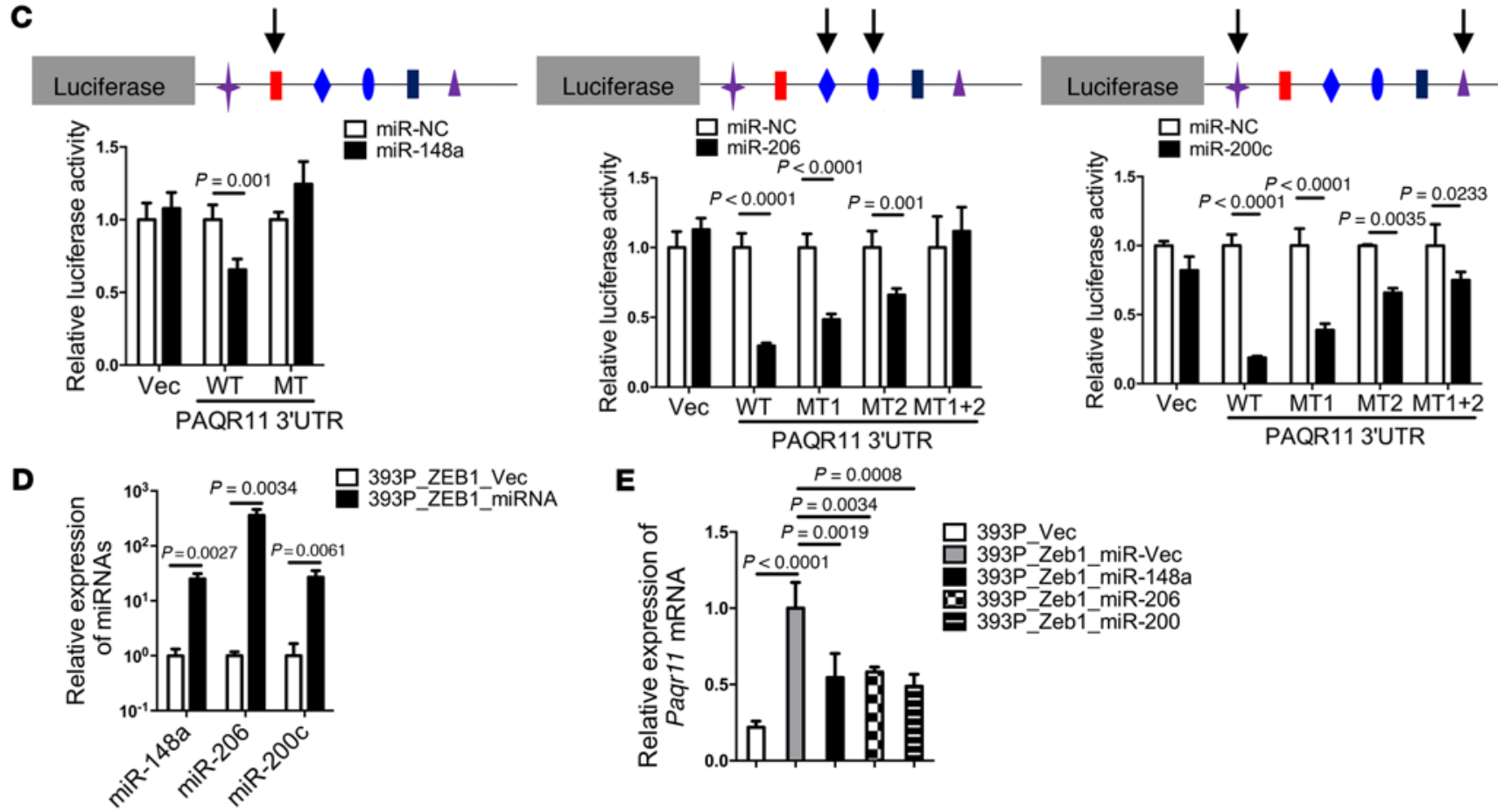

$\mathbf{F}$

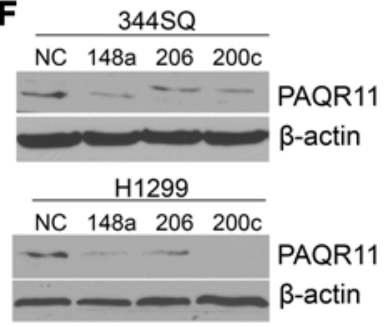

G

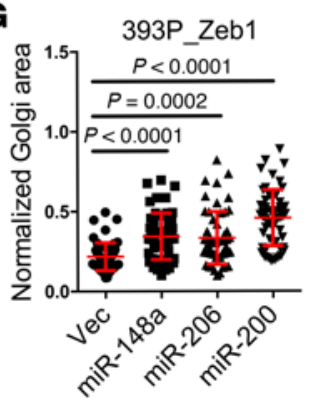

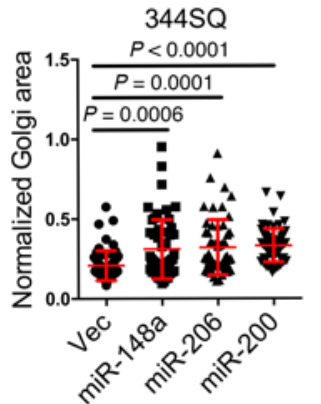

$\mathrm{H} 1299$

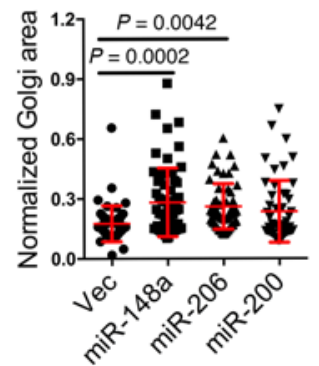

Figure 6. PAQR11 is a target of multiple miRs. (A) Heat map of PAQR11, ZEB1, and miR levels in KP cells ( $n=3$ samples per cell) with legend (left).

(B) The PAQR11 3'-UTR and predicted miR binding sites are shown at top. Bar graphs show luciferase activities in 344SQ cells (left) and H1299 cells (right) transiently cotransfected with PAQR11 $3^{\prime}$-UTR reporter and negative control (NC) or gene-specific pre-miRs $\left(n=3\right.$ samples per condition). ${ }^{\S} P<0.0001$.

(C) Illustrations of PAQR11 3'-UTR reporters (top) showing the mutated miR-148a site (left), mutated miR-206 sites (middle), and mutated miR-200c sites (right). Bar graphs show luciferase activities in 344SQ cells transiently cotransfected with negative control (NC) or gene-specific pre-miRs and reporters that were empty (Vec) or contained WT PAQR11 3'-UTR sequences or PAQR11 3'-UTRs with mutations in either (MT1 or MT2) or both (MT1+2) of the predicted binding sites. (D) The bar graph shows the results of qPCR analysis of ectopic miR-148a, -206, and -200c levels in 393P_ZEB1 cells stably transfected with vectors expressing ectopic miRs or with empty vectors (Vec). (E) The bar graph ( $n=3$ samples per condition) shows the results of qPCR analysis of $P A Q R 11$ mRNA levels in 393P_ZEB1 cells stably transfected with vectors expressing ectopic miRs or with empty vectors (Vec). (F) Western blot analysis of PAQR11 levels in 344SQ cells (top) and H1299 cells (bottom) transfected with pre-miRs or negative control (NC) oligos. $\beta$-Actin was included as loading control. (G) The scatter plots show Golgi areas normalized to nuclear areas in 393P_ZEB1 cells (left), 344SQ cells (middle), and H1299 cells (right) that stably express ectopic miRs or empty vector (Vec). Each dot represents values from a single cell. $P$ values were determined using 2-tailed Student's $t$ test or ANOVA for comparisons between 2 groups or more than 2 groups, respectively. Results were replicated ( $n \geq 2$ experiments). See complete unedited blots in the supplemental material. 
A

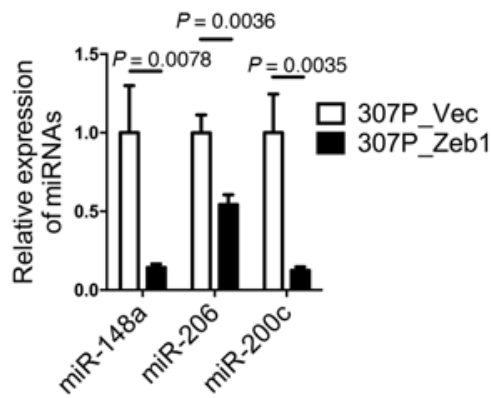

C

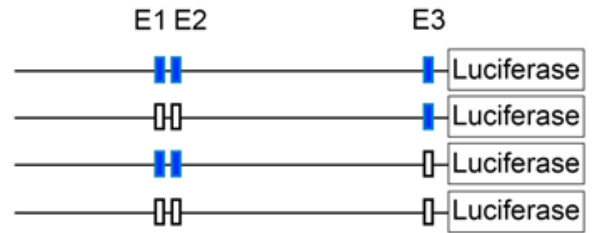

B

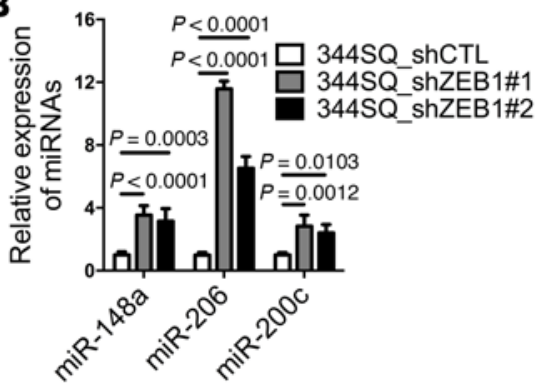

Relative luciferase activity

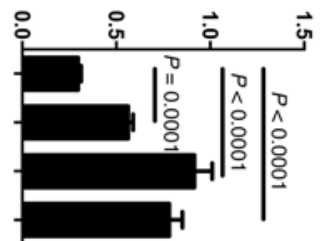

D

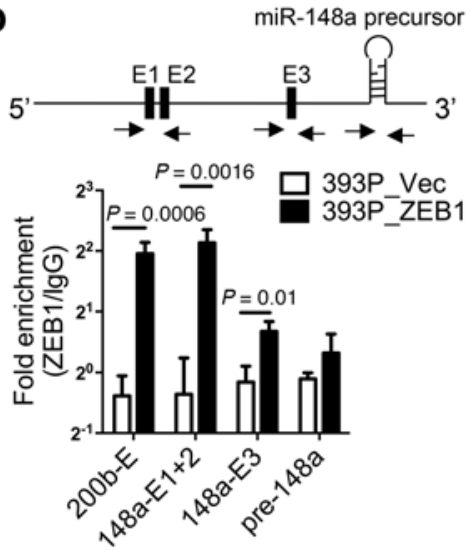

$\mathbf{E}$

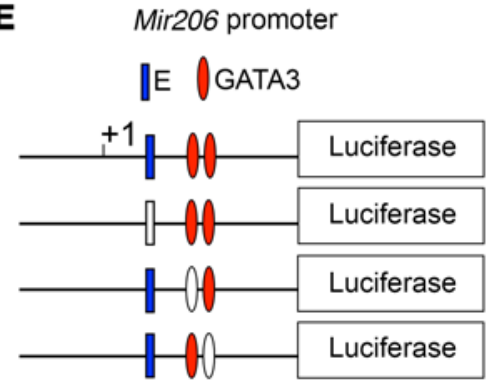

Relative luciferase activity

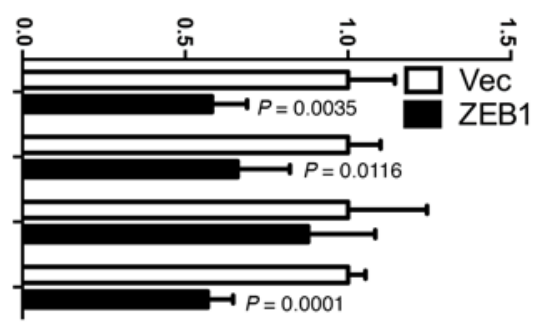

\section{保}

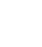
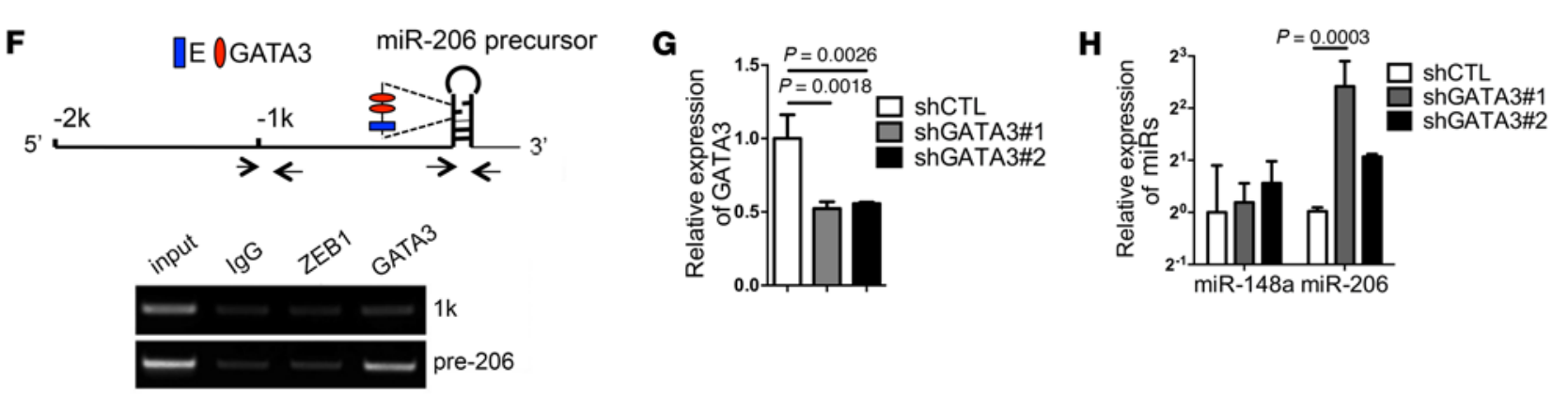

Figure 7. ZEB1 silences miR-206 and miR-148a through distinct mechanisms. (A) qPCR analysis of miR levels expressed as a ratio (ZEB1/Vec), with 307P_vector set at 1.0. (B) The bar graph shows qPCR analysis of miR levels in 344SQ cells stably transfected with ZEB1 shRNAs (\#1 or \#2) or scrambled control shRNA (shCTL). Results expressed as a ratio (shZEB1/shCTL), with 344SQ_shCTL set at 1.0. (C) Reporter activities in 393P cells transiently transfected with Mir148a promoter reporters containing WT (blue) or mutated (white) E-boxes. (D) miR-148a chromatin immunoprecipitation assays on chromatin immunoprecipitated with anti-ZEB1 or IgG antibodies. PCR amplification of E1/E2 locus (148a-E1+2) or E3 locus (148a-E3) on the Mir148a promoter. Results expressed as a ratio of qPCR values (anti-ZEB1/IgG). Mir200b/c/429 locus (200b-E) and the miR-148a precursor (pre-148a) were included as positive and negative controls, respectively. Arrows indicate locations of PCR primers. (E) Reporter activities in 393P_vector cells (Vec) and 393P_ZEB1 cells (ZEB1) transiently transfected with miR-206 promoter reporters containing intact (blue or red) or mutated (white) miR binding sites. (F) miR-206 ChIP assays. Chromatin was immunoprecipitated with anti-ZEB1 (ZEB1), anti-GATA3 (CATA3), or IgG antibodies. PCR amplification of sites within the miR-206 precursor (pre-206) or a negative control region in the miR-206 promoter (1k). Arrows indicate locations of PCR primers. (C) qPCR analysis of GATA3 levels in 344SQ cells stably transfected with GATA3 shRNA (shCATA3\#1 or shGATA3\#2) or scrambled shRNA (shCTL). Results are expressed as a ratio (shGATA3/ shCTL), with shCTL set at 1.0. (H) qPCR analysis of miR-148a levels and miR-206 levels. Results are expressed as a ratio (shCATA3/shCTL), with shCTL set at 1.0. Unless otherwise indicated, results represent the averages of triplicate samples per condition. $P$ values determined using 2 -tailed Student's $t$ test or ANOVA for comparisons between 2 groups or more than 2 groups, respectively. Results were replicated ( $n \geq 2$ experiments).

deficient 344SQ cells formed smaller but more numerous focal adhesions (Supplemental Figure 16A) and smaller lamellipodia (Supplemental Figure 16B), suggesting a defect in the maturation of these structures. However, PAQR11 depletion had minimal effects on tumor cell colony formation in soft agar (Supplemental
Figure 17A) or tumor cell proliferation in monolayer culture (Supplemental Figure 17B). To determine whether the miRs that target PAQR11 suppress tumor cell metastatic properties, we performed miR gain- and loss-of-function studies in KP cells. Ectopic expression of miR-148 or miR-206 suppressed the metastasis, but not 
A
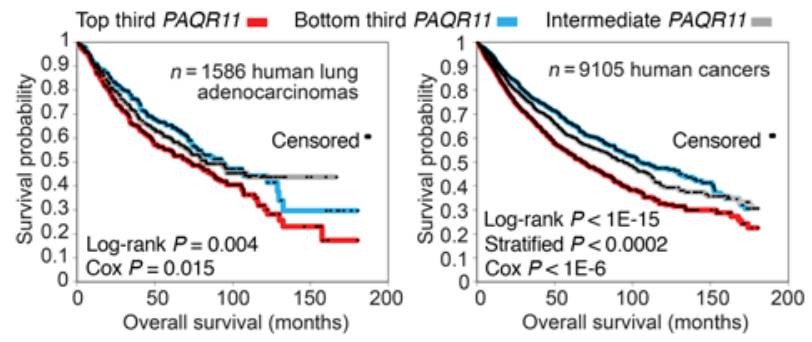

$\begin{array}{rrrrrrrr}\text { Top 530 } & 214 & 48 & 8 & \text { Top 3035 } & 519 & 112 & 28 \\ \text { Bottom 529 } & 246 & 41 & 7 & \text { Bottom 3035 } & 599 & 141 & 41 \\ \text { Inter. 527 } & 233 & 41 & 3 & \text { Inter. 3035 } & 599 & 147 & 39\end{array}$

C
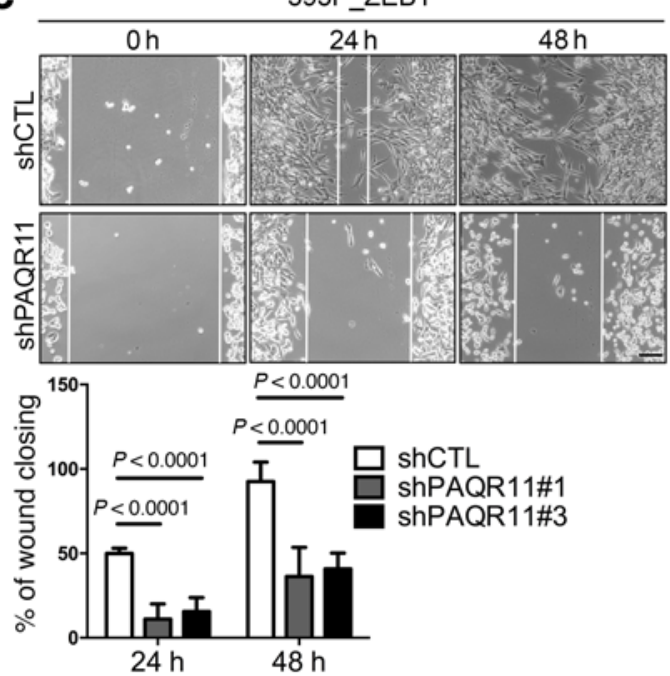

D

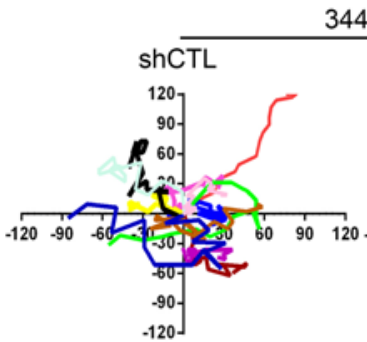

$\mathbf{E}$
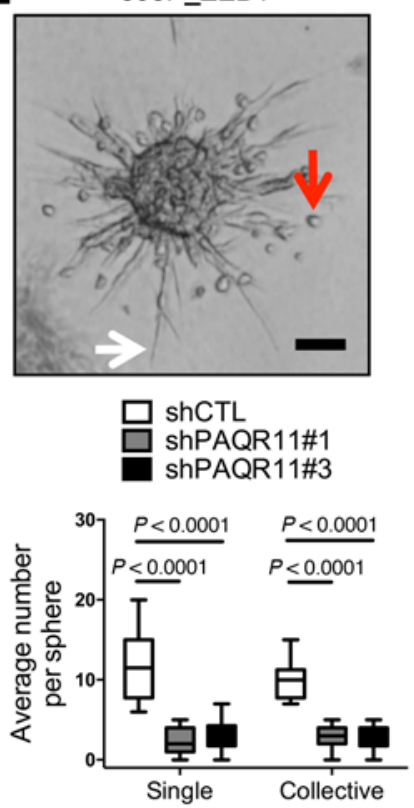

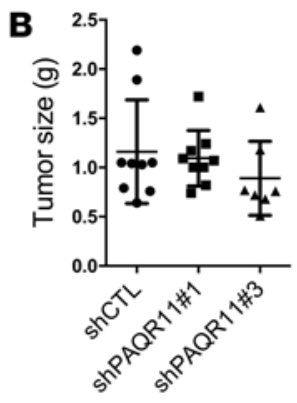

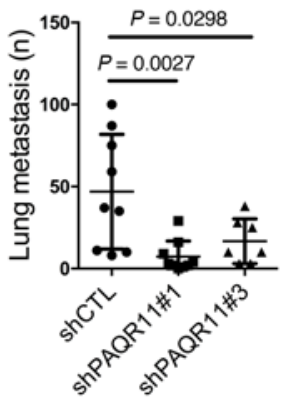

Figure 8. High PAQR11 levels enhance lung adenocarcinoma metastatic properties. (A) Kaplan-Meier plots of compendia of lung adenocarcinoma cohorts (left) and over 30 tumor types (right). The groups with highest ("top third"), lowest ("bottom third"), and intermediate ("middle third") PAQR11 mRNA levels were compared. Numbers of patients alive at each time point indicated under graphs. $P$ values determined by univariate Cox and stratified log-rank test correcting for cancer type. (B) Scatter plots of primary tumor weights (left) and lung metastasis numbers (right) per mouse (dots). (C) Brightfield micrographs of cells at the leading edges of scratch-wounded cultures of 393P_ZEB1 cells stably transfected with scrambled control shRNA (shCTL) or PAQR11 shRNA (shPAQR11). Bar graph shows percent wound closure 24 and 48 hours after placing of wounds. (D) Time-lapse tracing of movements of $344 \mathrm{SQ}$ cells stably transfected with scrambled control shRNA (shCTL) or PAQR11 shRNA (shPAQR11). Movements of individual cells over 6 hours indicated by colored lines ( $n=15$ cells per group). Bar graphs show quantification of cell speed (left) and directionality (right). (E) Bright-field micrograph of a 393P ZEB1 spheroid in collagen showing cells invading singly (red arrow) or collectively (white arrow). Scale bar: $100 \mu \mathrm{m}$. The bar and whisker plot shows quantification of single-cell (single) and collective invasions ( $n>20$ spheroids per group). (F) Bright-field micrographs of migrated and invaded cells in Boyden chambers. 393P_ZEB1 cells stably transfected with scrambled control shRNA (shCTL) or 1 of 2 distinct PAQR11 shRNAs (shPAQR11\#1 or shPAQR11\#3) stained with crystal violet (purple). Scale bar: $200 \mu \mathrm{m}$. Bar graph shows quantification of migrated and invaded cells. Unless otherwise indicated, results represent averages of triplicate samples per condition. $P$ values were determined using 2-tailed Student's $t$ test or ANOVA for comparisons between 2 groups or more than 2 groups, respectively. Results were replicated ( $n \geq 2$ experiments). 
the size, of 344SQ flank tumors in syngeneic, immunocompetent mice (Supplemental Figure 18A) and the migration and invasion of 393P_ZEB1 cells and 344SQ cells in Boyden chambers (Supplemental Figure 18, B and C), whereas antagomirs to miR-148a or miR-206 enhanced the migration and invasion of 393P cells (Supplemental Figure 18D). Collectively, these findings suggest that PAQR11 is a mediator of EMT-driven lung adenocarcinoma cell motility, invasion, and metastasis.

\section{Discussion}

Genome-wide copy number analyses and gain-of-function screens established GOLPH3 as an oncogene that is commonly targeted for amplification in human cancer (37). GOLPH3 bridges phosphatidylinositol-4-phosphate and actomyosin and thereby drives Golgi dispersal and vesicular budding $(3,38)$. A PITPNC1/ RAB1B protein complex anchors GOLPH3 to the Golgi, facilitating Golgi extension and the release of secretory vesicles containing factors that promote extracellular matrix remodeling and tumor angiogenesis (4). Collectively, these findings support a model in which pro-metastatic vesicular trafficking is driven by mechanical forces that promote Golgi dispersal.

In contrast to the GOLPH3-dependent mechanism by which metastasis occurs through Golgi dispersal, the findings presented here support an alternative model in which metastasis occurs through a Golgi compaction process that is accompanied by enhanced ribbon linking, cisternal stacking, and post-Golgi vesicular transport. This is analogous to the Golgi compaction and polarization observed in cells that undergo EMT and migrate directionally in wound healing assays $(39,40)$, implying that the pro-metastatic Golgi compaction process is part of a normal wound healing response that is co-opted by metastatic tumor cells in order to gain migratory and invasive properties.

The formation of ribbon-linked cisternal stacks is driven by several factors, including retrograde vesicle trafficking, which requires the tethering of retrograde vesicles to the Golgi by tethering factors such as the conserved oligomeric Golgi complex (COG), golgins (e.g., GM130 and GOLGIN-45), and the adhesive proteins GRASP55 and GRASP65 (29). During Drosophila spermatogenesis, COG and the Golgi-associated retrograde protein complex promote ribbon formation in the acroblast (41), and COG is essential for tethering activity in the cell-free vesicle tethering assay used here (32), implying that PAQR11 could well be acting as a scaffold in retrograde vesicle tethering, which in turn may be needed for ribbon formation. Additionally, both GRASP55 and GRASP65 are phosphorylated by ERK, a RAS-activated kinase, and mutation of the ERK phosphorylation sites on GRASP55 enhances cisternal stacking, indicating that ERK negatively regulates GRASP55 activity (42). PAQR11 recruits RAS proteins (H-, N-, and K-RAS) and RasGRP1 to the Golgi and is required for RAS-dependent ERK phosphorylation (25), arguing against RAS as a mediator of PAQR11-induced Golgi compaction. Instead, the findings presented here suggest that PAQR11 enables Golgi compaction by enhancing retrovesicle tethering to Golgi membranes and associating with multiple regulators of Golgi compaction and vesicle transport, scaffolding functions not previously attributed to PAQR11 or other PAQR family members.
Treatment of cells with brefeldin A and other inducers of Golgi stress such as golgicide A or Exo1 leads to Golgi fragmentation, inhibition of endoplasmic reticulum-Golgi transport, and impedance of protein secretion (43). Golgi fragmentation is a pathological feature of infection with Chlamydia trachomatis and Shigella flexneri and several neurodegenerative diseases, including amyotrophic lateral sclerosis, Alzheimer's, Parkinson's, and spinocerebellar ataxia type 2 (43-47). Golgi fragmentation by Golgi stress inducers has been largely ascribed to the inhibition of ARF small $G$ protein-guanine nucleotide exchange factor (GEF) complexes (43). ARF4 levels are upregulated by the CREB3 transcription factor following exposure to Golgi stress inducers, and ARF4 depletion confers resistance to stress-induced Golgi fragmentation (43). Thus, the Golgi actively senses stress stimuli and relays a signal to the nucleus to induce factors critical for the reestablishment of Golgi homeostasis in a manner akin to the unfolded protein response, a transcriptional program that maintains homeostasis in the endoplasmic reticulum following disturbances in cellular redox regulation or aberrantly high protein production (48). Whether Golgi stress response pathways also maintain Golgi integrity by upregulating PAQR11 levels through transcriptional programs like the one described here remains to be determined.

In one working hypothesis, tumor cells undergo EMT to disseminate from the primary site and revert to an epithelial state in the metastatic niche in order to colonize and proliferate $(49,50)$. The findings presented here raise the possibility that differentiation state plasticity requires transcriptional control of Golgi functions. However, lineage tracing studies in breast cancer models showed that metastatic tumor cells exhibit no evidence of having undergone EMT (51), and conditional deletion of transcriptional activators of EMT in pancreatic cancer models had no effect on metastasis (52), raising the possibility that EMT is entirely dispensable for metastasis or is relevant to metastasis in limited tumor types or specific mutational contexts. As shown here and elsewhere (53), metastatic tumor cells have the capacity to invade through collective or ameboid migratory modes, which may constitute a default pathway following genetic inactivation of EMT or a preferred pathway in certain tumor types or mutational contexts.

Inhibiting short- and long-range cellular migratory activities has the potential to suppress tumor growth and metastasis (54). Small-molecule inhibitors of kinesin motor proteins that control post-Golgi trafficking suppress tumor cell motility and inhibit mitosis in a broad spectrum of solid tumor types (55). The findings presented here highlight the potential utility of targeting processes in the Golgi to prevent metastasis in cancer patients.

\section{Methods}

See Supplemental Methods for full details.

Quantification of Golgi organelle area and Golgi element size and number. The area occupied by GM130-stained structures was quantified in volume projections with a limiting polygon. As described previously (56), the Golgi area was divided by the nucleus area in the same cell to generate a normalized area fraction ( $n=50-70$ cells per group). Cells were chosen randomly for such measurements. Golgi element size and number were determined from maximum intensity projections of deconvolved, thresholded (Otsu), and watershed-segmented 
3D stacks ( $n>50$ cells per group) by particle analysis (ImageJ, NIH; https://imagej.nih.gov/ij/).

FRAP assays. FRAP experiments were carried out as described previously $(57,58)$, with some modifications. Recovery was calculated within a circular region of interest (ROI) $1 \mu \mathrm{m}$ in diameter. Twenty prebleach images were acquired and averaged to estimate prebleach intensity. The laser power was set to cause greater than $70 \%$ bleach depth within 3 or 4 iterations. The first phase of post-bleach images were acquired at the highest speed to record the fast recovery (diffusion component) phase, and then 60-90 images were acquired at 1and 10-second intervals to record the slow recovery (binding component) phase. Time stacks were aligned by rigid body transformation and bleach-corrected using the average intensity from unbleached control cells. Recovery was calculated by the following equation:

$$
\frac{F_{s}-F_{b}}{F_{c}-F_{c_{0}}}
$$

(Equation 1)

where $F_{s}, F_{b}, F_{c}$, and $F_{c_{0}}$ represent the fluorescent intensities of the bleach ROI, background, control ROI before photobleaching, and control ROI after photobleaching, respectively. $F$ was expressed as the percentage of initial intensity. The data ( $n \geq 20$ cells from 4 independent experiments) were averaged (mean values \pm SEM) and, by nonlinear regression, fitted to the 1-phase exponential equation $Y=Y O+($ plateau $-Y 0) \times(1-\exp (-K \times x))$, where $Y O$ is the $Y$ value when $X$ (time) is 0 . The plateau is the $Y$ value at infinite times, and $K$ is the rate constant expressed in the reciprocal of seconds. The mobile fraction was given by the plateau, and the recovery half-time was computed as $T_{1 / 2}=\ln (2) / K$.

Golgi cisternal stack quantification. Electron microscopy was performed as previously described $(59,60)$. Sections of $60 \mathrm{~nm}$ were mounted on Formvar-coated nickel grids and double contrasted with $2 \%$ uranyl acetate for 5 minutes and 3\% lead citrate for 5 minutes. Grids were imaged using a Philips transmission electron microscope.

For analysis, Golgi stacks were identified using morphological criteria and quantified using standard stereological techniques $(59,60)$. Golgi images were captured at $\times 11,000$ magnification. The longest cisterna was measured as the cisternal length of a Golgi stack using the ruler tool in Photoshop CS6. At least 20 cells were quantified in each experiment, and the results represent 2 independent experiments.

VSV-G transport. The EGFP-VSV-G (ts045) plasmid (Addgene 11912) was a gift from Jennifer Lippincott-Schwartz (Janelia, Howard Hughes Medical Institute, Ashburn, Virginia, USA). As described previously (61), cells were transiently transfected with EGFP-VSV-G (ts045), transferred to the restrictive temperature of $40^{\circ} \mathrm{C}$ for 20 hours, and then transferred to the permissive temperature of $32^{\circ} \mathrm{C}$ for 1 hour, at which point the cells were fixed. In nonpermeabilized cells, exofacial and total VSV-G was detected by staining with an anti-VSV-G antibody and by measurement of EGFP signal intensity, respectively. Fluorescence intensity was quantified as described previously (62), and ratio images (exofacial to total) were generated using a modified version of methods described previously (63). The individual image channels were registered by rigid body transformation with a stack registration plugin (64). A total of 12-20 images per group were smoothed using a median filter and thresholded (Otsu). Pixel-by-pixel ratio images were generated using the follow- ing equation: (int $\mathrm{A}-\mathrm{bkgA}) /($ int $\mathrm{B}-\mathrm{bkg} \mathrm{B}) \times \mathrm{MF}$, where intR, int $\mathrm{A}$, and int $B$ are the intensities of the ratio image, first-channel images, and second-channel images, respectively, bkgA and bkgB are the background values of the corresponding images, and MF is an arbitrary multiplication factor. Pseudocolored images were generated by a custom gradient lookup table. For visualization purposes, the pseudocolored images were intensity-calibrated.

Cell-free assays of intra-Golgi vesicle tethering. Cell-free assays were performed as described (32). Briefly, cell pellets were resuspended in 4 times the pellet volume (1.5-2 ml) using $0.25 \mathrm{M}$ sucrose, $10 \mathrm{mM}$ HEPES pH 7.4, and lysed with 25 strokes in a tight-fitting Dounce homogenizer before floating of Golgi membranes following addition of 2.3 M sucrose and overlaying of the sample with $1.2 \mathrm{M}$ and $0.8 \mathrm{M}$ sucrose solutions buffered with HEPES. Golgi membranes collected from the $1.2 \mathrm{M} / 0.8 \mathrm{M}$ interface were immunoblotted for the myc tag present in the GalT-CFP reporter construct to ensure that equal amounts of Golgi were used for each sample. Assay mixes containing a standard amount of GalT-YFP vesicles, HEK293 cytosol, and energy regeneration system were incubated for 40 minutes either at $37^{\circ} \mathrm{C}$ or on ice, then imaged using epifluorescence, acquiring 13-16 images per temperature for each sample. Images were processed using ImageJ as described previously (65), and the percentage of colocalizing Golgi and vesicle particles was determined. The ratio of observed colocalizations at $37^{\circ} \mathrm{C}$ versus $0^{\circ} \mathrm{C}$ in each sample was normalized to a measurement done on the same day using Golgi membranes isolated from control and PAQR11-deficient HEK293 cells and was presented as an average of 4-5 experiments \pm SEM.

Microscopy. Confocal imaging was performed on a TCS-SP8 platform (Leica Microsystems) equipped with $\times 63 / 1.4 \mathrm{NA}$ oil, $\times 63 / 1.2 \mathrm{NA}$ water, or $\times 40 / 1.2$ NA oil objectives; 405/488/561/633 nm laser lines; and photomultiplier and hybrid (Leica HyD) detector systems. Highresolution images were acquired as described elsewhere $(57,66)$. For faster time-lapse imaging, an $8 \mathrm{~K}$ resonant scanner was used. For some live cell imaging experiments, an Ultraview spinning disc module (PerkinElmer) or a Nikon Eclipse Ti live cell imaging microscope was used. Wide-field fluorescence, bright-field, and phase-contrast imaging were performed on an IX71 microscope (Olympus). Images were subjected to iterative deconvolution (Huygens Professional, Scientific Volume Imaging) and processed using Image J. Background was subtracted using a rolling ball radius of 50-90 pixels. Colocalization analysis (Huygens Professional) was plotted using Manders' coefficients expressed as the percentage of red pixels that overlap with green pixels. Particle analysis routines (Image J) were used to measure focal adhesion parameters. Size of lamellipodia was estimated by selection of cortactin-stained lamellar structures using a free-hand selection macro (ImageJ).

Live and fixed cell imaging. Live cells were imaged in HEPESbuffered media within a cage incubator (Okolab). Golgi and PAQR11 time-lapse images were acquired at intervals of 2 minutes. Nuclei were counterstained with DRAQ5.

For fixed cell imaging, cells were fixed using $4 \%$ paraformaldehyde for 10 minutes, permeabilized using $0.1 \%$ Triton X-100 for 10 minutes, and blocked with 3\% BSA for 30 minutes. F-actin was detected using Alexa Fluor-conjugated phalloidin. Primary antibody incubation was performed in $3 \%$ BSA for 1 hour at room temperature or overnight at $4{ }^{\circ} \mathrm{C}$, followed by Alexa Fluor-conjugated secondary antibodies (1:500) in 3\% BSA for 1 hour at room temperature. Nuclei were detected with DAPI in cells on coverslips mounted in ProLong Gold Antifade mounting medi- 
um. Polarized spheres were fixed and stained as described previously (10). The staining of tumor tissue sections was carried out as described elsewhere (67). Line profiles were extracted from single-channel average intensity projections ( $n=12$ fields pooled from 3 tumors). Data were fitted with a single-phase exponential decay equation.

Statistics. Unless mentioned otherwise, the results shown are representative of replicated experiments and are the mean \pm SD from triplicate samples or randomly chosen cells within a field. Statistical evaluations were carried out with Prism 6 (GraphPad Software Inc.). Unpaired 2-tailed Student's $t$ tests were used to compare the mean values of 2 groups. ANOVA was used for analysis of parametric data. ANOVA with Dunnett's test was used for comparing multiple treatments to a control. ANOVA with a Bonferonni test was used when making multiple pair-wise comparisons between different groups. $P$ values of less than 0.05 were considered statistically significant. The Kaplan-Meier method with log-rank test was used to evaluate overall survival curves for patients from a pan-cancer analysis (6) and from a previously compiled compendium of independent lung adenocarcinoma cohorts (7). The latter includes expression profiling data sets for 11 previously reported human lung adenocarcinoma cohorts ( $n=1,492$ tumors) (7), with the addition of another data set from Sato et al. (68). Patients represented in both the Shedden and Chitale data sets ( $n=88$ patients) were first removed from the Shedden data set, and one patient from Bild data set thought to potentially represent SQCC was also removed (leaving $n=1,586$ tumors in total). For the pan-cancer analysis, we collected expression data on 9,105 tumors of various histological subtypes (ACC project, $n=$ 79; BLCA, $n=342$; BRCA, $n=1,072$; CESC, $n=299$; CHOL, $n=34$; COAD/READ, $n=632$; DLBC, $n=27$; GBM, $n=157$; HNSC, $n=506$; KICH, $n=65$; KIRC, $n=526$; KIRP, $n=256$; LAML, $n=160$; LGG, $n$ $=458$; LIHC, $n=353$; LUAD, $n=491$; LUSC, $n=480$; MESO, $n=71$; OV, $n=261$; PAAD, $n=163$; PCPG, $n=178$; PRAD, $n=436$; SARC, $n=243$; SKCM, $n=428$; TGCT, $n=121$; THCA, $n=498$; THYM, $n=$ 111; UCEC, $n=529$; UCS, $n=56$; UVM, $n=73$ ), for which RNA-seq data (v2 platform) and survival data were available, from The Cancer Genome Atlas(6). Patient survival was capped at 15 years.

Study approval. The present studies in animals were reviewed and approved by the Institutional Animal Care and Use Committee at The University of Texas MD Anderson Cancer Center in Houston, Texas.

\section{Author contributions}

JMK, XT, and PB conceived and designed the study. XT, PB, HFG, ARB, YW, DP, DLG, YZ, YX, and DU developed the methodology. JMK, XT, PB, SI, XL, YHA, HFG, JR, and IMN acquired data (provided animals, acquired and managed patients, provided facilities, etc.). XT, PB, CJC, and DU analyzed and interpreted data (e.g., statistical analysis, biostatistics, computational analysis). JMK, XT, PB, DLG, YW, and DU wrote, reviewed, and/ or revised the manuscript. JMK, XT, PB, CJC, XL, and TZ provided administrative, technical, or material support (i.e., reporting or organizing data, constructing databases). JMK provided study supervision.

\section{Acknowledgments}

We thank Yiqun Zhang, Bih-Fang Pan, and Xiaoyan Zhang for technical assistance. This work was supported by the NIH through R01 CA181184 (to JMK), R01 CA125123 (to CJC), GM087364 (to YW), GM105920 (to YW), GM112786P30 (to YW), EY007551 (to ARB), K08 CA151661 (to DLG), NRF-2010-0027945 (to YHA), and MD Anderson Cancer Center Support Grant CA016672; by the American Cancer Society through RGS-09-278-01-CSM (to YW); by Cancer Prevention Research Institute of Texas (CPRIT) Multi-investigator Research Award RP120713 (to JMK, CJC, and DLG); and by MCubed and the Fastforward Protein Folding Disease Initiative of the University of Michigan (to YW). JMK holds the Elza A. and Ina S. Freeman Endowed Professorship in Lung Cancer. DLG is an R. Lee Clark Fellow of The University of Texas MD Anderson Cancer Center supported by the Jeane F. Shelby Scholarship Fund. MD Anderson's Proteomics and Metabolomics Facility is supported by MD Anderson Cancer Center, NIH High-End Instrumentation Program grant 1S10OD012304-01, and CPRIT Core Facility grant RP130397. MD Anderson's South Campus Optical Microscopy Core is supported by NIH High-End Instrumentation Program grant 1S10RR029552-01.

Address correspondence to: Jonathan M. Kurie, MD Anderson Cancer Center, Box 432, Department of Thoracic/Head and Neck Medical Oncology, 1515 Holcombe Boulevard, Houston, Texas 77030, USA. Phone: 713.745.6747; E-mail: jkurie@mdanderson.org.
1. Goldenring JR. A central role for vesicle trafficking in epithelial neoplasia: intracellular highways to carcinogenesis. Nat Rev Cancer. 2013;13(11):813-820.

2. Yadav S, Linstedt AD. Golgi positioning. Cold Spring Harb Perspect Biol. 2011;3(5):a005322.

3. Dippold HC, et al. GOLPH3 bridges phosphatidylinositol-4- phosphate and actomyosin to stretch and shape the Golgi to promote budding. Cell. 2009;139(2):337-351.

4. Halberg N, Sengelaub CA, Navrazhina K, Molina H, Uryu K, Tavazoie SF. PITPNC1 Recruits RAB1B to the Golgi Network to Drive Malignant Secretion. Cancer Cell. 2016;29(3):339-353.

5. Buschman MD, Xing M, Field SJ. The GOLPH3 pathway regulates Golgi shape and function and is activated by DNA damage. Front Neurosci. 2015;9:362.

6. Brabletz T. EMT and MET in metastasis: where are the cancer stem cells? Cancer Cell. 2012;22(6):699-701.

7. Rodriguez-Boulan E, Macara IG. Organization and execution of the epithelial polarity programme. Nat Rev Mol Cell Biol. 2014;15(4):225-242.

8. Handler JS. Overview of epithelial polarity. Annu Rev Physiol. 1989;51:729-740.

9. Ahn $\mathrm{YH}$, et al. ZEB1 drives prometastatic actin cytoskeletal remodeling by downregulating miR-34a expression. J Clin Invest. 2012;122(9):3170-3183.

10. Gibbons DL, et al. Contextual extracellular cues promote tumor cell EMT and metastasis by regulating miR-200 family expression. Genes Dev. 2009;23(18):2140-2151.

11. Yang Y, et al. ZEB1 sensitizes lung adenocarcinoma to metastasis suppression by PI3K antagonism. J Clin Invest. 2014;124(6):2696-2708.

12. Lowe M, Gonatas NK, Warren G. The mitotic phosphorylation cycle of the cis-Golgi matrix protein GM130. J Cell Biol. 2000;149(2):341-356.

13. Kornfeld R, Kornfeld S. Assembly of asparagine-linked oligosaccharides. Annu Rev Biochem. 1985;54:631-664.

14. Ryan SD, et al. Microtubule stability, Golgi organization, and transport flux require dystonin-a2-MAP1B interaction. J Cell Biol. 2012;196(6):727-742.

15. Deakin NO, Turner CE. Paxillin inhibits HDAC6 to regulate microtubule acetylation, Golgi structure, and polarized migration. J Cell Biol. 2014;206(3):395-413.

16. Yang $Y$, et al. The Notch ligand Jagged 2 promotes lung adenocarcinoma metastasis through a miR-200-dependent pathway in mice. JClin Invest. 2011;121(4):1373-1385.

17. Kundu ST, et al. The miR-200 family and the miR-183 96 182 cluster target Foxf2 to inhibit 
invasion and metastasis in lung cancers. Oncogene. 2016;35(2):173-186.

18. Walton SM, et al. Spontaneous CD8 T cell responses against the melanocyte differentiation antigen RAB38/NY-MEL-1 in melanoma patients. JImmunol. 2006;177(11):8212-8218.

19. Wasmeier C, Romao M, Plowright L, Bennett DC, Raposo G, Seabra MC. Rab38 and Rab32 control post-Golgi trafficking of melanogenic enzymes. JCell Biol. 2006;175(2):271-281.

20. Yasuda T, Saegusa C, Kamakura S, Sumimoto H, Fukuda M. Rab27 effector Slp2-a transports the apical signaling molecule podocalyxin to the apical surface of MDCK II cells and regulates claudin-2 expression. Mol Biol Cell. 2012;23(16):3229-3239.

21. Torossi T, Roth J, Ziak M. A single tryptophan residue of endomannosidase is crucial for Golgi localization and in vivo activity. Cell Mol Life Sci. 2007;64(14):1881-1889.

22. Zuber C, Spiro MJ, Guhl B, Spiro RG, Roth J. Golgi apparatus immunolocalization of endomannosidase suggests post-endoplasmic reticulum glucose trimming: implications for quality control. Mol Biol Cell. 2000;11(12):4227-4240.

23. Pagano M, Jordan JD, Neves SR, Nguyen T, Iyengar R. Galphao/i-stimulated proteosomal degradation of RGS20: a mechanism for temporal integration of Gs and Gi pathways. Cell Signal. 2008;20(6):1190-1197.

24. Petry F, et al. Subcellular localization of rat Abca5, a rat ATP-binding-cassette transporter expressed in Leydig cells, and characterization of its splice variant apparently encoding a half-transporter. Biochem J. 2006;393(pt 1):79-87.

25. Jin T, et al. PAQR10 and PAQR11 mediate Ras signaling in the Golgi apparatus. Cell Res. 2012;22(4):661-676.

26. Liu L, et al. Proteomic characterization of the dynamic KSR-2 interactome, a signaling scaffold complex in MAPK pathway. Biochim Biophys Acta. 2009;1794(10):1485-1495.

27. Peng W, Lei Q, Jiang Z, Hu Z. Characterization of Golgi scaffold proteins and their roles in compartmentalizing cell signaling. J Mol Histol. 2014;45(4):435-445.

28. Yu X, Li Z, Chan MT, Wu WK. PAQR3: a novel tumor suppressor gene. Am J Cancer Res. 2015;5(9):2562-2568.

29. Puthenveedu MA, Bachert C, Puri S, Lanni F, Linstedt AD. GM130 and GRASP65-dependent lateral cisternal fusion allows uniform Golgi-enzyme distribution. Nat Cell Biol. 2006;8(3):238-248.

30. Xiang Y, Wang Y. GRASP55 and GRASP 65 play complementary and essential roles in Golgi cisternal stacking. JCell Biol. 2010;188(2):237-251.

31. Rabouille $\mathrm{C}$, Klumperman J. Opinion: The maturing role of COPI vesicles in intra-Golgi transport. Nat Rev Mol Cell Biol. 2005;6(10):812-817.

32. Cottam NP, Wilson KM, Ng BG, Körner C, Freeze $\mathrm{HH}$, Ungar D. Dissecting functions of the conserved oligomeric Golgi tethering complex using a cell-free assay. Traffic. 2014;15(1):12-21.

33. Luo X, et al. Characterization of the topology and functional domains of RKTG. Biochem J. 2008;414(3):399-406
34. Puisieux A, Brabletz T, Caramel J. Oncogenic roles of EMT-inducing transcription factors. Nat Cell Biol. 2014;16(6):488-494.

35. Brabletz S, Brabletz T. The ZEB/miR-200 feedback loop--a motor of cellular plasticity in development and cancer? EMBO Rep. 2010;11(9):670-677.

36. Creighton CJ, et al. Residual breast cancers after conventional therapy display mesenchymal as well as tumor-initiating features. Proc Natl Acad Sci U S A. 2009;106(33):13820-13825.

37. Scott KL, et al. GOLPH3 modulates mTOR signalling and rapamycin sensitivity in cancer. Nature. 2009;459(7250):1085-1090.

38. Farber-Katz SE, et al. DNA damage triggers Golgi dispersal via DNA-PK and GOLPH3. Cell. 2014;156(3):413-427.

39. Miller PM, Folkmann AW, Maia AR, Efimova N, Efimov A, Kaverina I. Golgi-derived CLASPdependent microtubules control Golgi organization and polarized trafficking in motile cells. Nat Cell Biol. 2009;11(9):1069-1080.

40. Darido C, Jane SM. Golgi Feels Its Own Wound. Adv Wound Care (New Rochelle). 2013;2(3):87-92.

41. Fári K, Takács S, Ungár D, Sinka R. The role of acroblast formation during Drosophila spermatogenesis. Biol Open. 2016;5(8):1102-1110.

42. Feinstein TN, Linstedt AD. Mitogen-activated protein kinase kinase 1-dependent Golgi unlinking occurs in $\mathrm{G} 2$ phase and promotes the G2/M cell cycle transition. Mol Biol Cell. 2007;18(2):594-604.

43. Reiling JH, et al. A CREB3-ARF4 signalling pathway mediates the response to Golgi stress and susceptibility to pathogens. Nat Cell Biol. 2013;15(12):1473-1485.

44. Sundaramoorthy V, Sultana JM, Atkin JD. Golgi fragmentation in amyotrophic lateral sclerosis, an overview of possible triggers and consequences. Front Neurosci. 2015;9:400.

45. Joshi G, Wang Y. Golgi defects enhance APP amyloidogenic processing in Alzheimer's disease. Bioessays. 2015;37(3):240-247.

46. Rendón WO, Martínez-Alonso E, Tomás M, Martínez-Martínez N, Martínez-Menárguez JA. Golgi fragmentation is Rab and SNARE dependent in cellular models of Parkinson's disease. Histochem Cell Biol. 2013;139(5):671-684.

47. Huynh DP, Yang HT, Vakharia H, Nguyen D, Pulst SM. Expansion of the polyQ repeat in ataxin-2 alters its Golgi localization, disrupts the Golgi complex and causes cell death. Hum Mol Genet. 2003;12(13):1485-1496.

48. Chevet E, Hetz C, Samali A. Endoplasmic reticulum stress-activated cell reprogramming in oncogenesis. Cancer Discov. 2015;5(6):586-597.

49. Ocaña OH, et al. Metastatic colonization requires the repression of the epithelial-mesenchymal transition inducer Prrx1. Cancer Cell. 2012;22(6):709-724.

50. Tsai JH, Donaher JL, Murphy DA, Chau S, Yang J. Spatiotemporal regulation of epithelial-mesenchymal transition is essential for squamous cell carcinoma metastasis. Cancer Cell. 2012;22(6):725-736.

51. Fischer KR, et al. Epithelial-to-mesenchymal transition is not required for lung metastasis but contributes to chemoresistance. Nature.
2015;527(7579):472-476.

52. Zheng X, et al. Epithelial-to-mesenchymal transition is dispensable for metastasis but induces chemoresistance in pancreatic cancer. Nature. 2015;527(7579):525-530.

53. Panková K, Rösel D, Novotný M, Brábek J. The molecular mechanisms of transition between mesenchymal and amoeboid invasiveness in tumor cells. Cell Mol Life Sci. 2010;67(1):63-71.

54. Waclaw B, Bozic I, Pittman ME, Hruban RH, Vogelstein B, Nowak MA. A spatial model predicts that dispersal and cell turnover limit intratumour heterogeneity. Nature. 2015;525(7568):261-264.

55. Purcell JW, et al. Activity of the kinesin spindle protein inhibitor ispinesib (SB-715992) in models of breast cancer. Clin Cancer Res. 2010;16(2):566-576.

56. Ng MM, Dippold HC, Buschman MD, Noakes CJ, Field SJ. GOLPH3L antagonizes GOLPH3 to determine Golgi morphology. Mol Biol Cell. 2013;24(6):796-808.

57. Banerjee P, Chander V, Bandyopadhyay A. Balancing functions of annexin A6 maintain equilibrium between hypertrophy and apoptosis in cardiomyocytes. Cell Death Dis. 2015;6:e1873.

58. Snapp EL, Altan N, Lippincott-Schwartz J. Measuring protein mobility by photobleaching GFP chimeras in living cells. Curr Protoc Cell Biol. 2003; Chapter 21:Unit 21.1.

59. Tang D, Xiang Y, Wang Y. Reconstitution of the cell cycle-regulated Golgi disassembly and reassembly in a cell-free system. Nat Protoc. 2010;5(4):758-772.

60. Wang Y, Satoh A, Warren G. Mapping the functional domains of the Golgi stacking factor GRASP65. J Biol Chem. 2005;280(6):4921-4928.

61. Starkuviene V, Seitz A, Erfle H, Pepperkok R. Measuring secretory membrane traffic: a quantitative fluorescence microscopy approach. Methods Mol Biol. 2008;457:193-201.

62. Gavet O, Pines J. Progressive activation of CyclinB1-Cdk1 coordinates entry to mitosis. Dev Cell. 2010;18(4):533-543.

63. Rudolf R, Mongillo M, Magalhães PJ, Pozzan T. In vivo monitoring of $\mathrm{Ca}(2+)$ uptake into mitochondria of mouse skeletal muscle during contraction. JCell Biol. 2004;166(4):527-536.

64. Thévenaz P, Ruttimann UE, Unser M. A pyramid approach to subpixel registration based on intensity. IEEE Trans Image Process. 1998;7(1):27-41.

65. Cossburn M, et al. Autoimmune disease after alemtuzumab treatment for multiple sclerosis in a multicenter cohort. Neurology. 2011;77(6):573-579.

66. Banerjee P, Bandyopadhyay A. Cytosolic dynamics of annexin A6 trigger feedback regulation of hypertrophy via atrial natriuretic peptide in cardiomyocytes. J Biol Chem. 2014;289(9):5371-5385.

67. Robertson D, Isacke CM. Multiple immunofluorescence labeling of formalin-fixed paraffin-embedded tissue. Methods Mol Biol. 2011;724:69-77.

68. Sato M, et al. Human lung epithelial cells progressed to malignancy through specific oncogenic manipulations. Mol Cancer Res. 2013;11(6):638-650. 